\title{
The richest superclusters
}

\section{Morphology}

\author{
M. Einasto ${ }^{1}$, E. Saar ${ }^{1}$, L. J. Liivamägi ${ }^{1}$, J. Einasto ${ }^{1}$, E. Tago ${ }^{1}$, V. J. Martínez ${ }^{2}$, J.-L. Starck ${ }^{3}$, \\ V. Müller ${ }^{4}$, P. Heinämäki ${ }^{5}$, P. Nurmi ${ }^{5}$, M. Gramann ${ }^{1}$, and G. Hütsi ${ }^{1}$ \\ 1 Tartu Observatory, 61602 Tõravere, Estonia \\ e-mail: maret@aai.ee \\ 2 Observatori Astronòmic, Universitat de València, Apartat de Correus 22085, 46071 València, Spain \\ 3 CEA-Saclay, DAPNIA/SEDI-SAP, Service d'Astrophysique, 91191 Gif-sur-Yvette, France \\ 4 Astrophysical Institute Potsdam, An der Sternwarte 16, 14482 Potsdam, Germany \\ 5 Turku University, Tuorla Observatory, Väisäläntie 20, Piikkiö, Finland
}

Received 8 June 2007 / Accepted 2 October 2007

\section{ABSTRACT}

Context. Superclusters are the largest systems in the Universe to give us information about the formation and evolution of structures in the very early Universe. Our present series of papers is devoted to the study of the morphology and internal structure of superclusters of galaxies.

Aims. We study the morphology of the richest superclusters from the catalogs of superclusters of galaxies in the 2dF Galaxy Redshift Survey and compare the morphology of real superclusters with model superclusters in the Millennium Simulation.

Methods. We use Minkowski functionals and shapefinders to quantify the morphology of superclusters: their sizes, shapes, and clumpiness. We generate empirical models of simple geometry to understand which morphologies correspond to the supercluster shapefinders (Appendix A).

Results. Rich superclusters have elongated, filamentary shapes with high-density clumps in their core regions. The clumpiness of superclusters is determined using the fourth Minkowski functional $V_{3}$. In the $K_{1}-K_{2}$ shapefinder plane the morphology of superclusters is described by a curve which is characteristic of multi-branching filaments as shown by our empirical models.

We found several differences between observed and model superclusters. The curves of the fourth Minkowski functional $V_{3}$ for observed and model superclusters have different shapes indicating that their structure is different. The values of $V_{3}$ for the supercluster SCL126 (the Sloan Great Wall) show that this supercluster has a very high density core which is absent in other superclusters. The values of the shapefinders $H_{1}-H_{3}$ and $K_{1}$ and $K_{2}$ for observed superclusters have much larger scatter than for model superclusters. The differences between the fourth Minkowski functional $V_{3}$ for the bright and faint galaxies in observed superclusters are larger than in simulated superclusters.

Conclusions. Our results show how the Minkowski functionals and shapefinders can be used to describe the morphology of superclusters: their shapes, sizes and clumpiness.

The shapes of observed superclusters are more diverse than the shapes of simulated superclusters. The larger scatter of the fourth Minkowski functional $V_{3}$ for the bright and faint galaxies for observed superclusters compared to simulated superclusters is an indication that the clumpiness of bright and faint galaxies in models does not reflect well the clumpiness of different galaxies in observed superclusters.

Our results suggest also that the volume covered by the Millennium Simulations may be too small to properly describe the large morphological variety of superclusters.

Key words. cosmology: large-scale structure of Universe - galaxies: clusters

\section{Introduction}

Superclusters of galaxies with characteristic dimensions of up to $100 \mathrm{~h}^{-1} \mathrm{Mpc}^{1}$ are the largest relatively isolated density enhancements in the Universe. Superclusters give us information about the early evolution of the structure in the Universe (Kofman et al. 1987) and about the properties of the supercluster-void network.

Early relatively deep all-sky catalogs of superclusters of galaxies were complied by Zucca et al. (1993) and Einasto et al. $(1994,1997,2001)$ on the basis of Abell clusters of galaxies (Abell 1958; Abell et al. 1989). New deep redshift surveys of galaxies (the Las Campanas Redshift Survey, the Sloan Digital

\footnotetext{
${ }^{1} h$ is the Hubble constant in units of $100 \mathrm{~km} \mathrm{~s}^{-1} \mathrm{Mpc}^{-1}$
}

Sky Survey and the 2 degree Field Galaxy Redshift Survey) cover large regions of sky and allow us to investigate the distribution of galaxies and the properties of galaxies up to rather large distances from us. These surveys have served as the basis for compiling catalogs of superclusters of galaxies (Einasto et al. 2003a,b; Basilakos 2003; and Erdogdu et al. 2004).

On the basis of the $2 \mathrm{dF}$ Galaxy Redshift Survey, we recently compiled a new catalog of superclusters of galaxies - Einasto et al. (2007a, hereafter Paper I). In this study we also compiled a catalog of superclusters of the Millennium Simulation by Springel et al. (2005), and used it to study possible selection effects. In Einasto et al. (2007b, hereafter Paper II) we studied the properties of superclusters. We characterized overall geometry of superclusters by their size and the degree of asymmetry 
and compactness, and compared them with similar parameters of simulated superclusters. In Einasto et al. (2007c, Paper III) we discussed properties of galaxies in superclusters, compared the density distributions and the properties of galaxy populations in rich and poor superclusters. We also compared the luminosity and multiplicity functions of observed and simulated superclusters (Einasto et al. 2006).

One of the goals of the forthcoming Planck mission is the study of the large scale structure using the Sunyaev-Zeldovich (SZ) effect. Cross-correlation of SZ selected and optically selected superclusters and rich superclusters in particular is part of the research which will be done using the Planck data.

In Papers I and II we showed that most properties of simulated superclusters and observed superclusters analyzed in these papers agree well with each other. This means that we can use the Millennium Simulation to generate superclusters for comparison with observations.

However, our studies showed that several differences exist between rich and poor superclusters. Rich superclusters contain high density cores, collections of rich galaxy clusters, poor groups and single galaxies, which are absent in poor superclusters (Paper III). Rich superclusters have a larger fraction of passive, red, non-star-forming galaxies than poor superclusters. Interestingly, we found that the fraction of very luminous superclusters in observed catalogs is larger than in simulated catalogs. Of course, it is possible that the Millennium Simulation, one realization of initial perturbations, may not be big enough to find a large number of very rich superclusters. However, its volume $\left(500 \mathrm{Mpc}^{3} h^{-3}\right)$ is three times larger than the total volume covered by our 2 dFGRS group catalog (Tago et al. 2006, hereafter T06), used to build the observed superclusters. Furthermore, we see rich superclusters in both halves of the 2dFGRS; this indicates that rich superclusters could be a typical feature of the galaxy distribution.

Therefore, among all superclusters, the richest superclusters deserve special attention. The richest relatively close superclusters are the Shapley Supercluster (Proust et al. 2006, and references therein) and the Horologium-Reticulum Supercluster (Rose et al. 2002; Fleenor et al. 2005; Einasto et al. 2003c). Very rich superclusters began to form earlier than other structures, they are sites of early star and galaxy formation (e.g. Mobasher et al. 2005) and first places where systems of galaxies form (e.g. Ouchi et al. 2004; Venemans et al. 2004). The supercluster environment affects the properties of groups and clusters located there (Plionis 2004). The fraction of X-ray clusters in rich superclusters is larger than in poor superclusters (Einasto et al. 2001), and the core regions of the richest superclusters may contain merging X-ray clusters (Rose et al. 2002; Bardelli et al. 2000).

In Paper II we presented a thorough review of earlier studies about the shapes and sizes of superclusters as well as a detailed comparison of sizes and shapes of rich and poor superclusters, using a number of parameters. Our study showed that the richest superclusters are more filamentary, less compact and more asymmetrical than poor superclusters.

One possibility to characterize the shape of an object was suggested by Sahni et al. (1998), who introduced shapefinders on the basis of Minkowski functionals. These shapefinders have been used before to estimate the filamentarity of superclusters (Sheth et al. 2003; Basilakos 2003).

In the present papers our goals are, first, to show how the morphology of individual superclusters can be quantified using the Minkowski functionals and shapefinders. Second, we apply these methods to study the morphology of the richest 2dFGRS superclusters and for comparison - the morphology of individual richest superclusters from the Millennium Simulation in detail (this paper) and to study substructures and galaxy populations in these richest superclusters (Einasto et al. 2007d, hereafter RII). This is the first time that the morphology and galaxy populations of observed superclusters is studied in detail, using the Minkowski functionals and shapefinders.

Therefore, in the present paper we use the Minkowski functionals and shapefinders to quantify the morphology of observed and simulated rich superclusters. In contrast to most earlier studies, we calculate the Minkowski functionals for the whole range of threshold densities, starting with the lowest density used in the supercluster search, up to the peak density in the supercluster core. We determine the clumpiness of superclusters using the fourth Minkowski functional $V_{3}$ and quantify the overall shape of superclusters by the $K_{1}-K_{2}$ shapefinder curves (the morphological signature). We also compare the Minkowski functionals of bright and faint galaxies. We generate a series of geometrical models which serve as prototypes of morphology to simulate the morphological signature of observed superclusters.

For our study we select the richest observed and simulated superclusters as described in Sect. 2.2.

The paper is composed as follows. In Sect. 2 we describe the galaxy data, the supercluster catalog and the data on the richest superclusters. In Sect. 3 we describe the Minkowski functionals and shapefinders used to study the morphology of superclusters, and present the results on supercluster morphology. In Sect. 4 we discuss our results and provide our conclusions. In the Appendix we introduce geometrical models as prototypes of morphology to study the shapefinders, and describe different kernels used to calculate the density fields of superclusters.

\section{Data}

\subsection{Catalogs of superclusters and groups}

We used the 2dFGRS final release (Colless et al. 2001, 2003) that contains 245591 galaxies. This survey has allowed the 2dFGRS Team and many other groups to estimate the fundamental cosmological parameters and to study intrinsic properties of galaxies in various cosmological environments; see Lahav (2004, 2005) for recent reviews. We used the data about galaxies and groups of galaxies (T06) to compile a catalog of superclusters of galaxies from the $2 \mathrm{dF}$ survey (Paper I). The $2 \mathrm{dF}$ sample becomes very diluted at large distances, thus we restrict our sample by a redshift limit $z=0.2$; we apply a lower limit $z \geq 0.009$ to avoid confusion with unclassified objects and stars. When calculating (comoving) distances we use a flat cosmological model with the standard parameters: matter density $\Omega_{\mathrm{m}}=0.3$, dark energy density $\Omega_{\Lambda}=0.7$ (both in units of the critical cosmological density).

Galaxies were included in the 2dFGRS, if their corrected apparent magnitude $b_{j}$ lied in the interval from $m_{1}=13.5$ to $m_{2}=19.45$. The faint limit actually fluctuates from field to field. Our aim is to restore as well as possible the actual luminosity density, accounting also for galaxies outside the survey magnitude limits (for details see T06 and Paper III). Therefore these fluctuations have been taken into account in the calculation of weights assigned to galaxies, needed to find the luminosity density field. The weights are proportional to the ratio of the expected total luminosity to the luminosity in the observational window of the survey at the distance of the galaxy.

In the calculation of weights we used, for every galaxy, the individual values of the faint end magnitudes of the observational window, $m_{2}$. We also used a correction for the 
incompleteness factor $c=\gamma(1-\exp (m-\mu))$, where $\gamma=0.99$, $m$ is the observed magnitude of the galaxy, and the parameter $\mu$ varies from field to field (see Eq. (5) of Colless et al. 2001). The weight of the galaxy is proportional to the inverse of the incompleteness factor. To calculate weights, we assumed that galaxy luminosities are distributed according to the Schechter (1976) luminosity function.

We used the weighted luminosities of galaxies to calculate the luminosity density field on a grid with cell size of $1 h^{-1} \mathrm{Mpc}$ and smoothed with an Epanechnikov kernel of radius $8 h^{-1} \mathrm{Mpc}$ (see Appendix B for details); this density field was used to find superclusters of galaxies. We defined superclusters as connected non-percolating systems with densities above a certain threshold density; the actual threshold density used was 4.6 in units of the mean luminosity density. A detailed description of the supercluster finding algorithm can be found in Paper I.

Later we shall use the data on the luminosities of galaxies to divide galaxies by their luminosity into the populations of bright and faint galaxies. We wanted to use an absolute magnitude limit close to the break luminosity $M^{\star}$ in the Schechter luminosity function. According to the estimates of the luminosity function, the value of $M^{\star}$ is different for different galaxy populations (Madgwick et al. 2003a; de Propris et al. 2003; Croton et al. 2005); having values from -19.0 to $-20.9^{2}$. Therefore we used a bright/faint galaxy limit $M_{b j}=-20.0$ as a compromise between the different values (see also Paper III).

For comparison we used simulated galaxy samples of the Millennium Simulation by Springel et al. (2005). This simulation was made using a very large number of dark matter particles $\left(2160^{3}\right)$ in a periodic box of $500 \mathrm{~h}^{-1} \mathrm{Mpc}$, and adopting standard values of cosmological parameters. For identifying superclusters in simulations, we adopted the same selection window as in the case of observed superclusters (Paper I). Using semi-analytic methods, catalogs of simulated galaxies were calculated by Croton et al. (2006). The simulated galaxy catalog contains almost 9 million objects, for which positions and velocities are given, as well as absolute magnitudes in the Sloan Photometric system $(u, g, r, i, z)$. The limiting absolute magnitude of the catalog is -17.4 in the $r$ band.

Our analysis in Paper I showed that, in general, the properties of superclusters from the Millennium Simulation and the 2dFGRS sample agree well. We did not find systematic differences between the observed and model superclusters which could be caused by the use of different photometric systems ( $2 \mathrm{dF}$ versus SDSS for Millennium).

The catalogs of observed groups and isolated galaxies can be found at http://www . aai .ee/ maret/2dfgr.html, the catalogs of observed and model superclusters - at http: //www . aai . ee/ maret/2dfscl .html.

\subsection{The richest superclusters in real and simulated catalogs}

Since our present analysis is focused on the study of the richest superclusters, we selected from both our catalogs four very rich superclusters. From the $2 \mathrm{dF}$ superclusters we chose, firstly, the two richest superclusters, one from the Northern and one from the Southern sky. These are the richest superclusters in our catalog: the supercluster SCL126 in the Northern sky, and the supercluster SCL9 (the Sculptor supercluster) in the Southern sky, according to the catalog by Einasto et al. (2001, hereafter E01).

The other two observed superclusters were selected as follows. There are several rich superclusters in the 2dFGRS

\footnotetext{
${ }^{2}$ All absolute magnitudes have been calculated for $h=1$.
}

supercluster catalog, but they are far away, and due to the magnitude limit of the survey, we would only get a small number of galaxies in volume-limited supercluster galaxy samples. As we wanted to study the galaxy content of the superclusters in an accompanying paper, we had to select rich, but also relatively nearby superclusters. In the Northern sky we selected the supercluster SCL88 (Sextans); this supercluster is the third richest in the Northern sky, according to the number of galaxies, but we chose this supercluster because it is relatively nearby and thus includes fainter galaxies than more distant rich superclusters. In the Southern sky we chose the nearby supercluster SCL10 (the Pisces-Cetus supercluster, this is also the second richest supercluster in the Southern sky according to the number of galaxies). All these superclusters are only partly covered by the $2 \mathrm{dF}$ Survey region (E01; Einasto et al. 2003c).

A description of these superclusters is given in Table 1. There we provide the coordinates and distances of superclusters, the numbers of galaxies, groups and Abell and X-ray clusters in the superclusters, the mean values of the luminosity density field in the superclusters and their total luminosities (from Paper II). In our analysis we use volume-limited samples of galaxies from these superclusters. The luminosity limits for these samples for each supercluster are also given in Table 1.

The most prominent Abell supercluster in the Northern $2 \mathrm{dF}$ survey is the supercluster SCL126 (in E01, N152 in Paper I) in the direction of the Virgo constellation. This supercluster has also been called the Sloan Great Wall (Vogeley et al. 2004; Gott et al. 2005; Nichol et al. 2006).

Another rich supercluster in the Northern Sky is the Sextans supercluster, SCL88 (in E01; N20 in Paper I). Only a small part of this supercluster is located inside the $2 \mathrm{dF}$ survey volume, including one of seven Abell clusters from this supercluster.

The richest supercluster in the Southern Sky is the Sculptor supercluster (SCL9 in E01; S34). There are several X-ray clusters in this supercluster. This supercluster contains the largest number of Abell clusters in our supercluster sample, 25. However, only 12 of them are located in the region covered by the $2 \mathrm{dF}$ redshift survey.

Another nearby prominent supercluster in the Southern sky is the Pisces-Cetus supercluster (SCL10 in E01, S5 in Paper I) which contains the rich X-ray cluster, Abell 2734. Only one of 19 Abell clusters from this supercluster is located inside the $2 \mathrm{dF}$ survey boundaries. This supercluster was recently described as a rich filament of Abell clusters by Porter \& Raychaudhury (2005).

From the Millennium simulation, we also selected the richest superclusters. We first use the data on the three richest superclusters. The supercluster M3 is the ninth richest in the catalog by the number of galaxies, but the reason to include this system in our analysis is that this supercluster is the second richest by the number of density field (DF) clusters in it.

We define DF clusters (see Paper II) as local maxima of the luminosity density; they correspond to observed galaxy clusters. In Paper II we divided superclusters into richness classes, using the number of DF clusters in them as a quantitative richness indicator. Superclusters with 1 or 2 DF clusters are classified as poor, and those with more than $10 \mathrm{DF}$ clusters as rich superclusters (the large number of DF clusters was the reason why we included the simulated supercluster M3 in our analysis, see the previous paragraph).

Most superclusters in our catalog are poor. Thus as a morphological template for poor superclusters we use the data on the best-known nearby supercluster, the Local Supercluster, denoted as V20 due to the chosen distance limit. The Local 
Table 1. Data on rich superclusters.

\begin{tabular}{|c|c|c|c|c|c|c|c|c|c|c|c|c|}
\hline$\overline{\mathrm{ID}}$ & $\begin{array}{l}\text { RA } \\
\text { deg }\end{array}$ & $\begin{array}{l}\text { Dec } \\
\text { deg }\end{array}$ & $\begin{array}{r}D \\
h^{-1} \mathrm{Mpc}\end{array}$ & $\overline{N_{\mathrm{gal}}}$ & $\overline{M_{\mathrm{lim}}}$ & $\overline{N_{\mathrm{vol}}}$ & $\overline{N_{\mathrm{cl}}}$ & $\overline{N_{\mathrm{gr}}}$ & 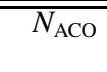 & $\overline{\overline{N_{\mathrm{X}}}}$ & $\overline{\overline{\delta_{\mathrm{m}}}}$ & $\overline{L_{\text {tot }}}$ \\
\hline SCL88 (20) & 155.11 & -2.54 & 184.5 & 556 & -17.50 & 484 & $\overline{2}$ & 7 & $1(7)$ & 2 & 5.7 & $0.319 \mathrm{E}+13$ \\
\hline SCL126 (152) & 194.71 & -1.74 & 251.2 & 3591 & -19.25 & 1308 & 18 & 40,2 & 9 & 5 & 7.7 & $0.378 E+14$ \\
\hline SCL10 (5) & 1.85 & -28.06 & 177.4 & 952 & -17.50 & 757 & 5 & 5 & $1(19)$ & 5 & 6.2 & $0.482 E+13$ \\
\hline SCL9 (34) & 9.85 & -28.94 & 326.3 & 3175 & -19.50 & 1176 & 24 & 26,9 & $12(25)$ & 6 & 8.1 & $0.497 \mathrm{E}+14$ \\
\hline M1 (195) & & & & 5437 & -19.25 & 1589 & 9 & & & & 8.2 & $0.204 E+14$ \\
\hline M2 (1089) & & & & 5047 & -19.5 & 4048 & 25 & & & & 7.4 & $0.489 E+14$ \\
\hline M3 (1386) & & & & 2016 & -19.5 & 2007 & 17 & & & & 7.2 & $0.638 E+14$ \\
\hline M4 (207) & & & & 3645 & -19.5 & 1794 & 9 & & & & 7.8 & $0.581 E+14$ \\
\hline
\end{tabular}

Note: Identity ID is according to Einasto et al. (2001) with the name of Paper I in parenthesis; with sky coordinates and distance $D$ for our cosmology; the galaxy number $N_{\text {gal }}$ for the whole superclusters; the magnitude limit $M_{\text {lim }}$ and the galaxy number $N_{\text {vol }}$ for volume limited parts of the supercluster galaxies; $N_{\mathrm{cl}}$ and $N_{\mathrm{gr}}$ are density field cluster and group numbers according to Paper I; $N_{\mathrm{ACO}}$ and $N_{\mathrm{X}}$ gives the number of Abell and X-ray clusters, respectively, in that part of the supercluster covered by $2 \mathrm{dF}$ survey, the number inside parenthesis is the total number of Abell clusters in this supercluster by Einasto et al. (2001) list; $\delta_{\mathrm{m}}$ - the mean values of the luminosity density field in superclusters, in units of mean density; $L_{\text {tot }}-$ supercluster total luminosity in Solar units.
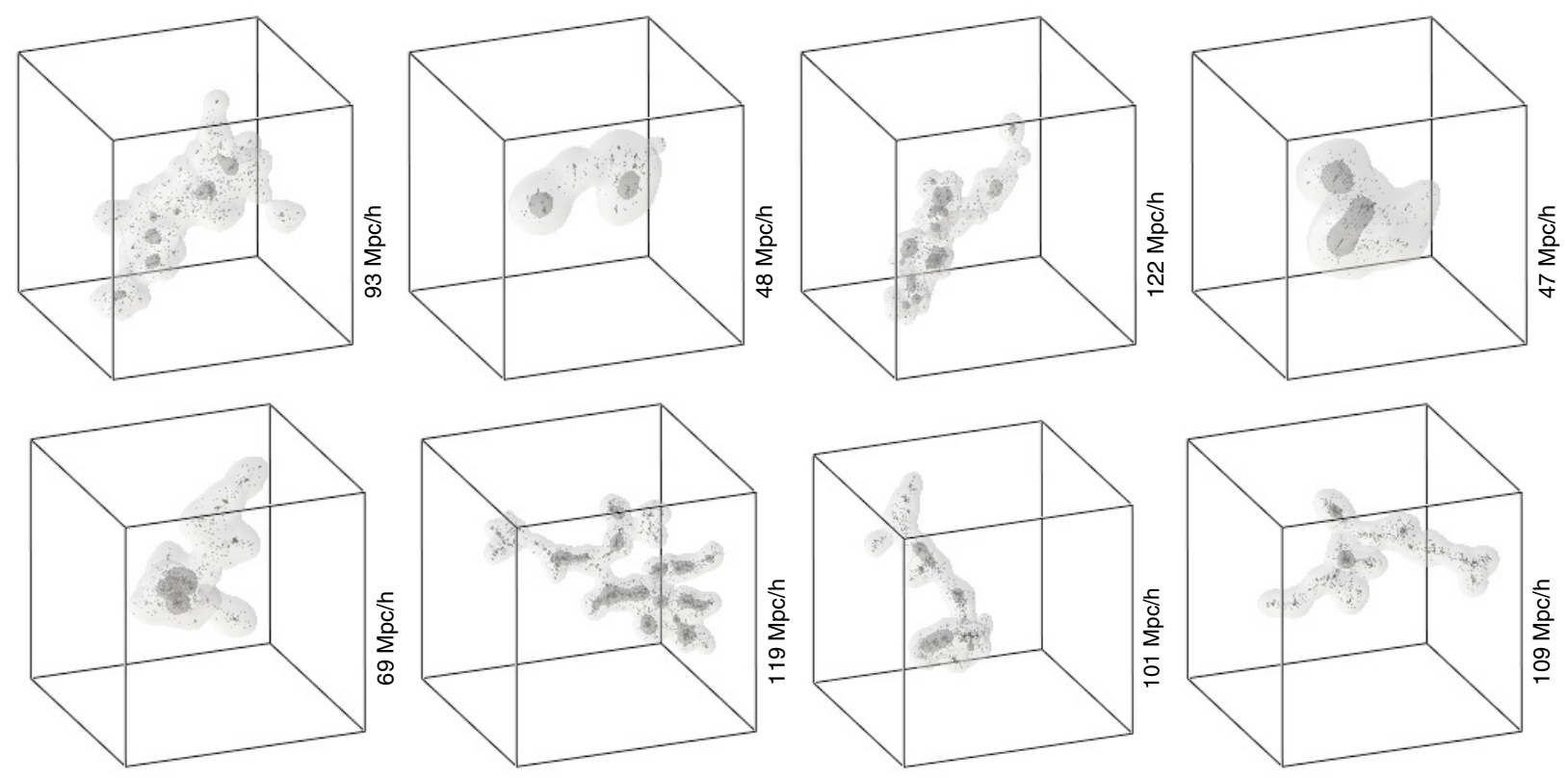

Fig. 1. The density field view of the superclusters. The light gray region shows the full supercluster, and the dark gray shows the density level for the central (core) region which corresponds to the maximum value of the Minkowski functional $V_{3}$ (the richest substructure). This region contains about 0.4-0.1 of the total mass of the supercluster (see Table 2). Galaxies are shown with small dots. Upper panels from left to right: SCL126, SCL88, SCL9, SCL10. Lower panels from left to right: M1, M2, M3, M4.

Supercluster represents a typical poor supercluster of a rather small size, with one rich galaxy cluster, the Virgo cluster, in the center, surrounded by filaments of galaxies and poor groups. The Local Group is located near the edge of the supercluster. The total luminosity of the Local Supercluster is $L=3 \times 10^{12} h^{-2} L_{\odot}$, and its mass is $M=1 \times 10^{15} h^{-1} M_{\odot}$. Most superclusters in our catalog of the 2dFGRS superclusters are of the Local Supercluster type (Paper I). The data on the Local Supercluster galaxies are taken from ZCAT (http://www.cfa.harvard.edu/ huchra/zcat/). In total we have in this supercluster 328 galaxies in a volume-limited sample $(M \leq-18.0)$, with the maximum distance of $20 h^{-1} \mathrm{Mpc}$.

The distribution of galaxies in regions of different density in real and simulated superclusters is shown in Fig. 1. We see at a first glance how filamentary all the superclusters are. The presence of several concentration centers, as well as many highdensity knots is also clearly seen. Historically, the first paper to show quantitatively the filamentary nature of superclusters was by Einasto et al. (1984) using the friend-of-friends method. The filamentary structure of superclusters is not a kernel effect, as the kernels used in density estimation are isotropic. However, the lengths of these filaments (supercluster sizes) depend on the choice of the kernel size. See Sect. 3.2 and Appendix B for the description of our choices; see also a detailed discussion in Paper I.

\section{Morphology of superclusters}

\subsection{Morphological descriptors}

We characterize superclusters by their outer (isodensity) surface, and its enclosed volume. When increasing the isodensity level over the threshold overdensity, we move into the central parts of the supercluster. The morphology and topology of the isodensity 
Table 2. The Minkowski functionals and shapefinders for rich superclusters.

\begin{tabular}{rrrrrrrrrrrr}
\hline \hline ID & $m_{\mathrm{f}}$ & $V_{0}$ & $V_{1}$ & $V_{2}$ & $V_{3}$ & $H_{1}(\mathrm{~T})$ & $H_{2}(\mathrm{~W})$ & $H_{3}(\mathrm{~L})$ & $K_{1}(\mathrm{P})$ & $K_{2}(\mathrm{~F})$ & $K_{1} / K_{2}$ \\
\hline SCL126 & 0.0 & $1.27 \mathrm{e} 5$ & 2566.0 & 56.9 & 1 & 24.67 & 28.71 & 42.67 & 0.08 & 0.20 & 0.39 \\
& 0.89 & 1462 & 210.2 & 41.2 & 9 & 3.48 & 3.25 & 30.91 & -0.03 & 0.81 & -0.04 \\
SCL88 & 0.0 & $2.84 \mathrm{e} 4$ & 743.10 & 24.44 & 1 & 19.11 & 19.36 & 18.33 & 0.01 & -0.03 & $-2.36 \mathrm{e}-01$ \\
& 0.65 & 927 & 103.40 & 15.33 & 3 & 4.48 & 4.29 & 11.50 & -0.02 & 0.46 & $-4.71 \mathrm{e}-02$ \\
SCL9 & 0.0 & $1.75 \mathrm{e} 5$ & 3591.0 & 78.11 & 1 & 24.42 & 29.27 & 58.58 & 0.09 & 0.33 & 0.27 \\
& 0.79 & 3855 & 445.3 & 71.11 & 15 & 4.33 & 3.99 & 53.33 & -0.04 & 0.86 & -0.05 \\
SCL10 & 0.0 & $3.72 \mathrm{e} 4$ & 885.80 & 27.11 & 1 & 20.97 & 20.80 & 20.33 & -0.004 & -0.01 & 0.35 \\
& 0.86 & 425 & 59.56 & 11.33 & 3 & 3.57 & 3.35 & 8.50 & -0.03 & 0.43 & -0.07 \\
& & & & & & & & & & & \\
V20 & 0.0 & $3.05 \mathrm{e} 4$ & 736.90 & 23.33 & 1 & 20.72 & 20.11 & 17.50 & -0.02 & -0.07 & 0.22 \\
& 0.43 & 1589 & 121.60 & 11.56 & 2 & 6.53 & 6.70 & 8.67 & 0.01 & 0.13 & 0.10 \\
& & & & & & & & & & & \\
M1 & 0.0 & $7.29 \mathrm{e} 4$ & 1649.00 & 42.56 & 1 & 22.09 & 24.67 & 31.92 & 0.06 & 0.13 & 0.43 \\
& 0.59 & 2354 & 244.00 & 33.89 & 5 & 4.82 & 4.58 & 25.42 & -0.03 & 0.69 & -0.04 \\
& & & & & & & & & & & \\
M2 & 0.0 & $1.82 \mathrm{e} 5$ & 3772.0 & 77.78 & 1 & 24.18 & 30.87 & 58.34 & 0.12 & 0.31 & 0.40 \\
& 0.65 & 5930 & 644.0 & 91.89 & 15 & 4.60 & 4.46 & 68.92 & -0.02 & 0.88 & -0.018 \\
M3 & 0.0 & $1.19 \mathrm{e} 5$ & 2624.0 & 63.67 & 1 & 22.64 & 26.24 & 47.75 & 0.07 & 0.29 & 0.25 \\
& 0.65 & 3860 & 420.9 & 60.00 & 9 & 4.59 & 4.47 & 45.00 & -0.01 & 0.82 & -0.02 \\
M4 & 0.0 & $1.06 \mathrm{e} 5$ & 2381.00 & 58.00 & 1 & 22.26 & 26.13 & 43.50 & 0.08 & 0.25 & 0.32 \\
& 0.60 & 3454 & 374.90 & 55.78 & 9 & 4.61 & 4.28 & 41.84 & -0.04 & 0.81 & -0.05 \\
\hline
\end{tabular}

Columns: Col. 1: Supercluster ID, Col. 2: mass fraction, $m_{\mathrm{f}}$, Cols. 3-6: Minkowski functionals $V_{0}-V_{3}, V_{0}$ in $\left(h^{-1} \mathrm{Mpc}\right)^{3}, V_{1}$ in $\left(h^{-1} \mathrm{Mpc}\right)^{2}, V_{2}$ in $h^{-1} \mathrm{Mpc}$, Cols. 7-9: shapefinders $H_{1}$ (thickness), $H_{2}$ (width) and $H_{3}$ (length), in $h^{-1} \mathrm{Mpc}$, Cols. 10-12: shapefinders $K_{1}$ (planarity), $K_{2}$ (filamentarity) and their ratio, $K_{1} / K_{2}$.

contours is (in the sense of global geometry) completely characterized by four Minkowski functionals.

For a given surface the four Minkowski functionals are:

1. the first Minkowski functional $V_{0}$ is the enclosed volume $V$,

2. the second Minkowski functional $V_{1}$ is proportional to the area of the surface $S$, namely,

$$
V_{1}=\frac{1}{6} S
$$

3. the third Minkowski functional $V_{2}$ is proportional to the integrated mean curvature $C$,

$V_{2}=\frac{1}{3 \pi} C, \quad C=\frac{1}{2} \int_{S}\left(\frac{1}{R_{1}}+\frac{1}{R_{2}}\right) \mathrm{d} S$,

where $R_{1}$ and $R_{2}$ are the two local principal radii of curvature;

4. the fourth Minkowski functional $V_{3}$ is proportional to the integrated Gaussian curvature (or Euler characteristic) $\chi$,

$V_{3}=\frac{1}{2} \chi, \quad \chi=\frac{1}{2 \pi} \int_{S}\left(\frac{1}{R_{1} R_{2}}\right) \mathrm{d} S$.

The Euler characteristic is simply related to the genus, $G$

$G=1-V_{3}$

The fourth Minkowski functional gives us the number of isolated clumps (or voids) in the sample (Martinez et al. 2005; Saar et al. 2007). One should beware of extra factors of 2 that are sometimes seen in formulae as (4). With conventional normalization there should be no extra factors in (4).
To characterize the shape of an object Sahni et al. (1998) and Shandarin et al. (2004) introduced shapefinders, a set of combinations of Minkowski functionals: $H_{1}=3 \mathrm{~V} / \mathrm{S}$ (thickness), $H_{2}=S / C$ (width), and $H_{3}=C / 4 \pi$ (length). These quantities have dimensions of length and are normalized to give $H_{i}=R$ for a sphere of radius $R$. For a convex surface, the shapefinders $H_{i}$ follow the inequalities $H_{1} \leq H_{2} \leq H_{3}$. Oblate ellipsoids (pancakes) are characterized by $H_{1} \ll H_{2} \approx H_{3}$, while prolate ellipsoids (filaments) are described by $H_{1} \approx H_{2} \ll H_{3}$.

Additionally, Sahni et al. (1998) defined two dimensionless shapefinders $K_{1}$ (planarity) and $K_{2}$ (filamentarity): $K_{1}=\left(H_{2}-\right.$ $\left.H_{1}\right) /\left(H_{2}+H_{1}\right)$ and $K_{2}=\left(H_{3}-H_{2}\right) /\left(H_{3}+H_{2}\right)$.

Then, after Sahni et al. (1998), the following shapes can be distinguished:

1. spheres with $H_{1}=H_{2}=H_{3}$, i.e. $K_{1}=K_{2}=0$;

2. ideal filaments with $K_{1} \approx 0, K_{2} \approx 1$;

3. real filaments with $K_{1}<K_{2}$;

4. ideal pancakes with $K_{1} \approx 1, K_{2} \approx 0$;

5. pancakes with $K_{1}>K_{2}$;

6. ideal ribbons with $K_{1} \approx K_{2} \approx 1$;

7. ribbons with $K_{1} / K_{2} \approx 1$.

In the $\left(K_{1}, K_{2}\right)$-plane filaments are located near the $K_{2}$-axis, pancakes near the $K_{1}$-axis, and ribbons along the diagonal, connecting the spheres at the origin with the ideal ribbon at $(1,1)$.

\subsection{Supercluster morphology}

We present the results of our calculations of Minkowski functionals and shapefinders in Table 2 and Figs. 2-4. The original luminosity density field, used to delineate superclusters (Papers I, II), was calculated using all galaxies. For 

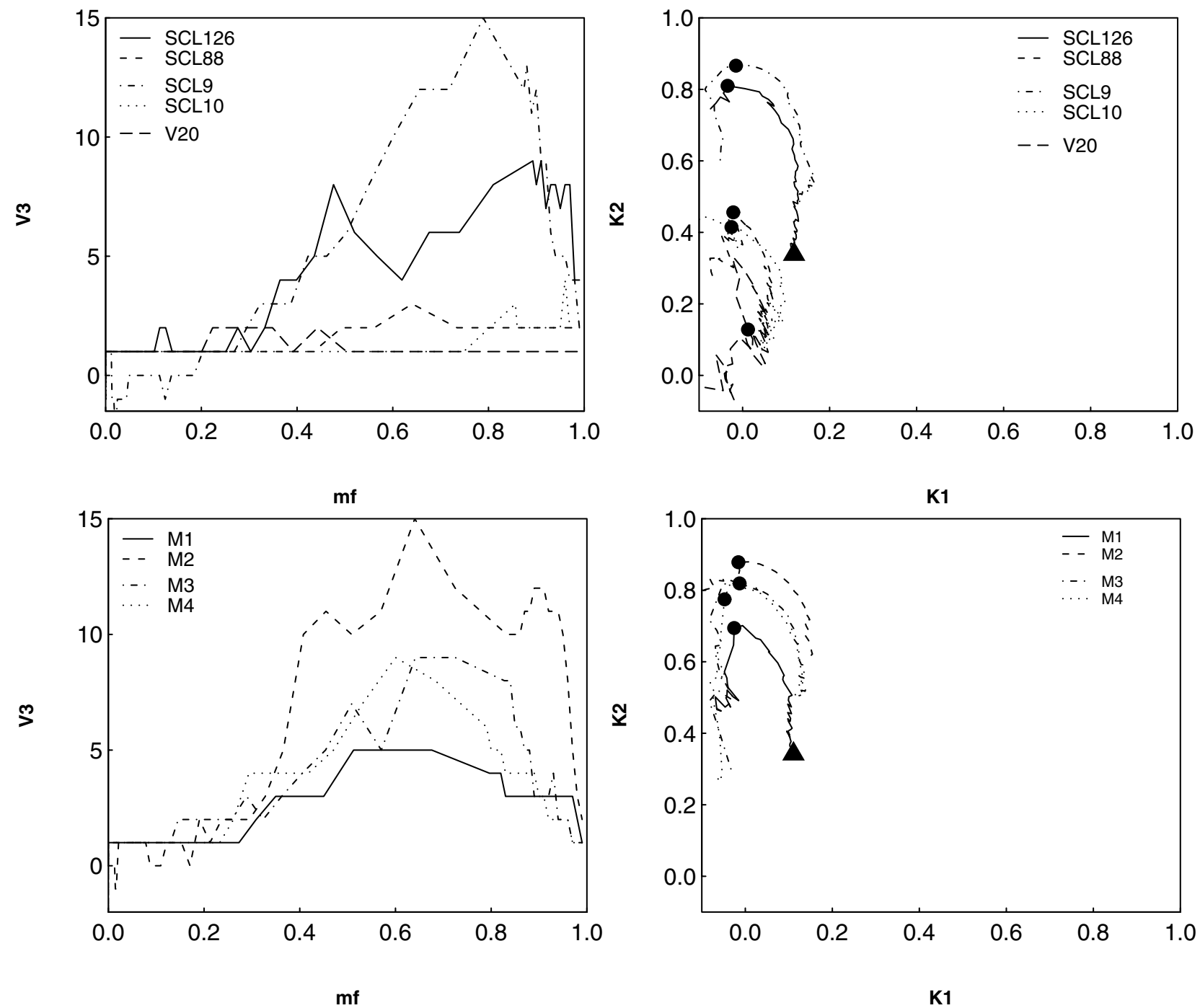

Fig. 2. The Minkowski functional $V_{3}$ (the Euler characteristic) (left panels) and the shapefinders $K_{1}$ (planarity) and $K_{2}$ (filamentarity) (right panels) for the observed (upper panels) and simulated (lower panels) superclusters. In the right panels we indicate for SCL126 and M1 with triangles the values of $K_{1}, K_{2}$, where the mass fraction $m_{\mathrm{f}}=0.0$ (the whole supercluster), and for all superclusters with filled circles the values of $K_{1}, K_{2}$, which correspond to the $m_{\mathrm{f}}$, at which $V_{3}$ has a maximum.

morphological studies we have to use volume-limited galaxy samples; this makes our results insensitive to selection corrections. For this reason the density field had to be recalculated. We used a kernel estimator with a $B_{3}$ box spline as the smoothing kernel, with the total extent of $16 h^{-1} \mathrm{Mpc}$ (for a detailed description see Appendix and Saar et al. 2007). This kernel covers exactly the $16 h^{-1} \mathrm{Mpc}$ extent of the Epanechnikov kernel, used to obtain the original density field, but it is smoother and resolves better density field details (its effective width is about $8 h^{-1} \mathrm{Mpc}$ ). As the argument labeling the isodensity surfaces, we chose the (excluded) mass fraction $m_{\mathrm{f}}$ - the ratio of the mass in regions with density lower than the density at the surface, to the total mass of the supercluster. When this ratio runs from 0 to 1 , the iso-surfaces move from the outer limiting boundary into the center of the supercluster, i.e. the fraction $m_{\mathrm{f}}=0$ corresponds to the whole supercluster, and $m_{\mathrm{f}}=1$ to its highest density peak. This definition may seem unnatural, but we kept it as it is in accordance with that used in all topological studies of the largescale structure to date (see, e.g., Sheth et al. 2003; Saar et al. 2007, and references therein). The reason for this convention (the higher the density level, the higher the value of the mass fraction) is historical - the most popular argument for the genus and for the Minkowski functionals has been a volume fraction that grows with the density level; all other arguments are chosen to run in the same direction.

In Table 2 we give for all superclusters the values of the Minkowski functionals and shapefinders for two mass fraction values: $m_{\mathrm{f}}=0.0$, which corresponds to the whole supercluster, and the value of $m_{\mathrm{f}}$, at which the fourth Minkowski functional $V_{3}$ has a maximum (except for SCL126, see below) - this shows the maximum number of isolated cores (clumps) that the supercluster breaks into. At lower densities (mass fractions) these clumps are joined together, and at higher densities they start to disappear, when the density levels get higher than their maximum density.

\subsubsection{Minkowski functionals for superclusters}

At small mass fractions the iso-density surface includes the whole supercluster. Thus volumes and areas of superclusters ( $V_{0}$ and $V_{1}$, Table 2) are large. As we move to higher mass 

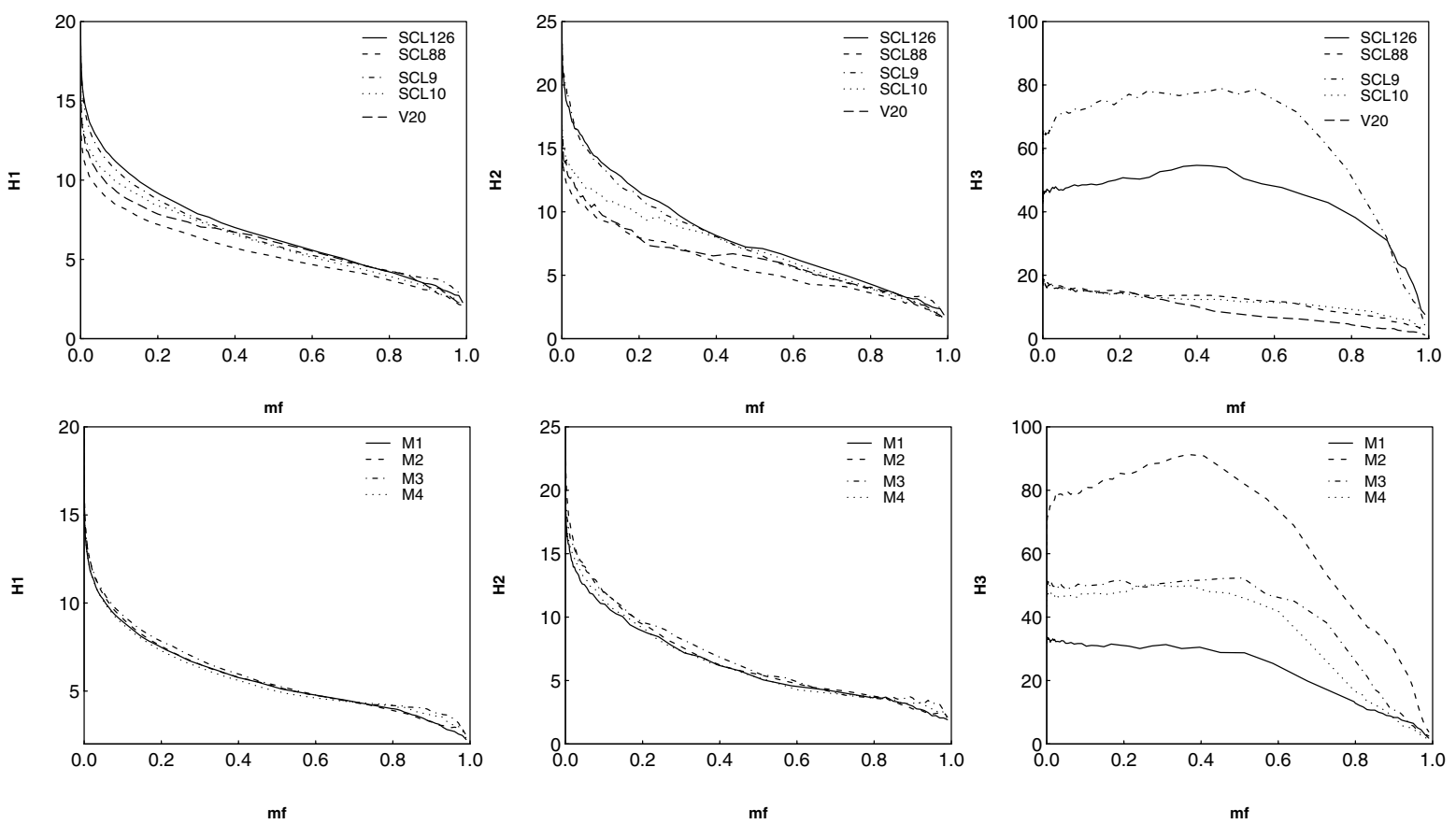

Fig. 3. Shapefinders $H_{1}$ (thickness), $H_{2}$ (width), $H_{3}$ (length) (in $h^{-1} \mathrm{Mpc}$ ) for the observed superclusters (upper panels) and for the simulated superclusters (lower panels) versus the mass fraction $m_{\mathrm{f}}$.
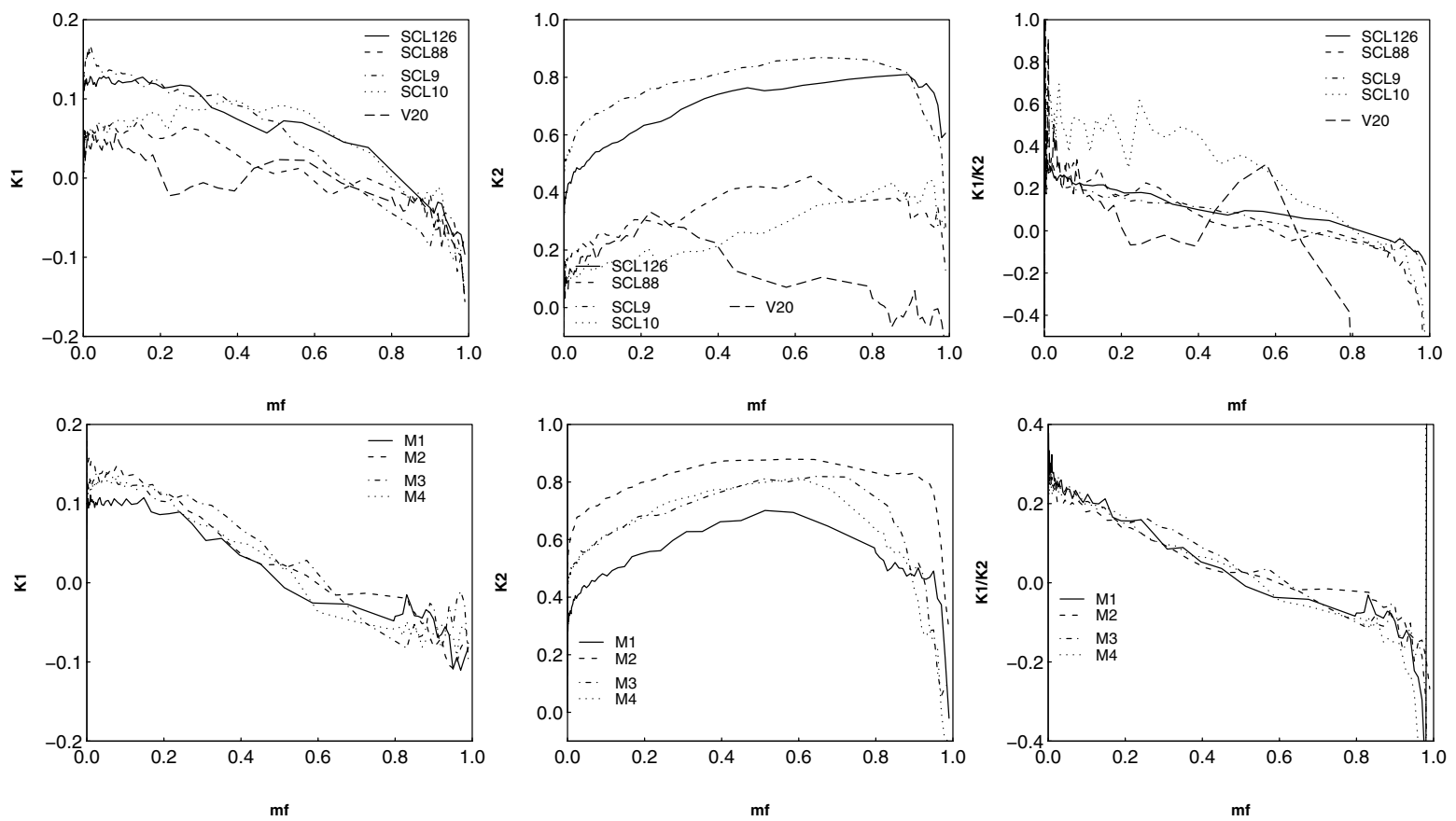

Fig. 4. Shapefinders $K_{1}$ (planarity) and $K_{2}$ (filamentarity) for the observed superclusters (upper panels) and for the simulated superclusters (lower panels) versus the mass fraction $m_{\mathrm{f}}$.

fractions, the iso-density surfaces include only higher density parts of superclusters, and their volumes and areas get smaller. At very high mass fractions only the highest density clumps in superclusters give their contribution to the supercluster. Individual high density regions in a supercluster, which at low mass fraction are joined together into one system, began to separate from each other, and the value of the fourth Minkowski functional $\left(V_{3}\right)$ increases. At a certain density contrast (mass fraction) $V_{3}$ has a maximum showing the largest number of isolated clumps in a given supercluster at the spatial resolution determined by the smoothing kernel. At still higher density contrasts only the highest density peaks contribute to the supercluster.

Figure 2 (left panels) shows the fourth Minkowski functional $V_{3}$ for the most massive real and simulated superclusters. The $V_{3}$ curve for the supercluster SCL9 in the left upper panel shows a characteristic behavior. At the mass fraction value of about 0.2 , the value of $V_{3}$ for SCL9 begins to increase and reaches a maximum value at $m_{\mathrm{f}} \approx 0.75$. The values of $V_{3}$ then begin to decrease. This indicates that the overall morphology of the supercluster SCL9 is clumpy; this supercluster consists of a large number of clumps or cores connected by relatively thin 
filaments, in which the density of galaxies is too low to contribute to the supercluster center over a certain mass fraction. The maximum value of the fourth Minkowski functional $V_{3}$ (Table 2) shows that the supercluster SCL9 has the largest number of isolated clumps, although this supercluster is only partly covered by our sample - the value of $V_{3}$ for the whole supercluster may be twice as large as our present calculations show. This supercluster is the largest and richest of observed superclusters in our present sample, with the largest size and volume.

The second richest and largest supercluster among the observed superclusters is the supercluster SCL126. Figure 2 shows that the $V_{3}$ curve for SCL126 has a shape which is quite different from that for SCL9. At a mass fraction $m_{\mathrm{f}} \approx 0.4$ the value of $V_{3}$ increases rapidly, peaks, decreases again and has another peak at a mass fraction of about $m_{\mathrm{f}} \approx 0.85$. This behavior indicates that the overall morphology of the supercluster SCL126 is quite homogeneous, which is characteristic of a rich filament with several branches (see also Sect. 3.3). Interestingly, the $V_{3}$ curve for the supercluster SCL126 shows several peaks at a high mass fraction, $m_{\mathrm{f}}>0.95$. This indicates the presence of a very high density core region with several individual clumps in it - this is the main core region of the supercluster with several Abell clusters, which are also X-ray clusters (Einasto et al. 2003c). In other superclusters we do not see such a high density and very compact core. Another example of a supercluster with a high density core is the simulated supercluster M2, but in this supercluster the peaks at high mass fraction in the $V_{3}$ curve appear at mass fractions $m_{\mathrm{f}}<0.95$.

In Fig. 2 the observed superclusters separate clearly into two different classes. The superclusters SCL88 and SCL10 have much smaller numbers of density peaks than the superclusters SCL126 and SCL9. This may be partly explained by the incompleteness of the SCL10 and SCL88, which are cut by the survey boundaries. In the case of the Virgo supercluster V20 the maximum value of the fourth Minkowski functional is only 2, describing a compact supercluster.

The shapes of the $V_{3}$ curves for simulated superclusters in Fig. 2 and their maximum values show large variations. In the case of the supercluster M2 the $V_{3}$ curve shows a rapid increase at the mass fraction $m_{\mathrm{f}} \approx 0.4$, and three maxima, one at $m_{\mathrm{f}} \approx 0.9$ showing that this supercluster is very clumpy. The maximum value of $V_{3}$ is comparable to that for the observed supercluster SCL9, and the presence of a peak at high values of $m_{\mathrm{f}}$ is comparable to that for SCL126. However, the mass fraction at which the peak occurs is lower than in the case of SCL126.

The shapes of the $V_{3}$ curves for simulated superclusters M3 and M4 resemble those for the observed supercluster SCL9; however, the maximum values of $V_{3}$ are less than 10 , showing that the number of isolated cores or clumps in these simulated superclusters is smaller than in SCL9. The simulated supercluster M1 has the smallest maximum value of $V_{3}$ among the selected simulated superclusters showing that this supercluster is less clumpy than other simulated superclusters. The number of isolated clumps in this supercluster is still larger than that for the observed superclusters SCL10 and SCL88.

We have determined the number of density field clusters in superclusters (Paper I). Tables 1 and 2 show that in all superclusters except SCL88 and M4 the maximum value of $V_{3}$ is about half that density field clusters indicating that typically high density cores in superclusters contain two density field clusters. In superclusters SCL88 and M4 these values are equal. The value of $V_{3}$ for SCL9 and M2 is negative at the mass fraction $m_{\mathrm{f}} \approx 0$, indicating that there are holes through the supercluster.
Using the data in Table 2 we can estimate that in high density regions of superclusters (at mass fractions where the value of the fourth Minkowski functional has a maximum) the density of galaxies is about ten times higher than the mean density of galaxies in the same supercluster.

In summary, in this subsection we showed how the Minkowski functionals characterize the volume, area and clumpiness of a supercluster. We quantified the clumpiness of a supercluster by the fourth Minkowski functional $V_{3}$ which determines the number of isolated cores or clumps in superclusters. The shape of the $V_{3}\left(m_{\mathrm{f}}\right)$ curve gives us information about the overall morphology of a supercluster. We showed that the supercluster SCL126 resembles a rich filament with several branches, while other rich superclusters can be described as having a large number of clumps connected by chains of galaxies ("multispiders"). The maximum values of the fourth Minkowski functional $V_{3}$ for the richest superclusters from observations and from models (SCL9 and M2) coincide $\left(V_{3}^{\max }=15\right)$. However, the shapes of the $V_{3}\left(m_{\mathrm{f}}\right)$ curves for these superclusters are different, indicating that the structure of these superclusters differs, the supercluster M2 having a larger number of clumps for a wide range of mass fraction values.

We note that in the Millennium Simulation we use the SDSS $r$-band luminosities, i.e. more strongly clustered red galaxies should dominate. This could lead to systematically larger numbers of clumps at high mass fraction values for model superclusters and, as a result, systematically larger values of the fourth Minkowski functional $V_{3}$ than in observed superclusters. Figure 2 shows that this is not the case. Therefore the differences between model and observed superclusters are not due to the different photometric systems.

\subsubsection{Shapefinders $H_{1}-H_{3}$}

Next we analyze the shapefinders $H_{1}-H_{3}$ for superclusters. These quantities have dimensions of length, and $H_{1} \leq H_{2} \leq H_{3}$ in the case of a convex body (e.g., triaxial ellipsoid). Therefore, they can be used to study the dimensions of superclusters. The shapefinder $H_{1}$ is the smallest and characterizes the thickness of superclusters. The shapefinder $\mathrm{H}_{2}$ as an intermediate one is an analogy of the width of a supercluster. The width is calculated as $H_{2}=S / C$, and contains information about both the area and curvature of an isodensity surface. The shapefinder $\mathrm{H}_{3}$ is the longest and describes the length of the superclusters. Of course, this is not the real length of the supercluster, but a measure of the integrated curvature of the surface which may become very large for irregularly shaped and curved surfaces.

In Fig. 3 (upper panels) we plot the shapefinders $H_{1}$ to $H_{3}$ for the richest superclusters. This figure shows that the extension of the superclusters as measured by the shapefinders $H_{1}$ and $H_{2}$ is about $15-20 h^{-1} \mathrm{Mpc}$ for the complete supercluster $\left(m_{\mathrm{f}}=0\right.$, Table 2). At higher mass fractions, $m_{\mathrm{f}} \approx 0.5$, the iso-surfaces include only higher density parts of superclusters, $H_{1}$ and $H_{2}$ are less than $10 h^{-1} \mathrm{Mpc}$, i.e. the supercluster centers are still typical 3-dimensional objects. At mass fractions which correspond to the maximum value of $V_{3}$ (the core regions of superclusters) $H_{1}$ and $H_{2}$ are about $5 h^{-1} \mathrm{Mpc}$. The scatter of the shapefinder $H_{2}$ for observed superclusters is larger than that of $H_{1}$ showing the influence of a different number of substructures (isolated clumps or cores) in these systems.

The shapefinder $\mathrm{H}_{3}$ differs strongly for the four observed superclusters. For the whole superclusters $\left(m_{\mathrm{f}}=0\right)$ the values of $\mathrm{H}_{3}$ are about $20 \mathrm{~h}^{-1} \mathrm{Mpc}$ for poorer superclusters and 40-60 $h^{-1} \mathrm{Mpc}$ for the two richest superclusters, SCL126 and 
SCL9 (Table 2). We note that for the richest two superclusters at mean mass fractions $m_{\mathrm{f}} \approx 0.5$ the value of $\mathrm{H}_{3}$ is larger than at $m_{\mathrm{f}}=0$, reaching a maximum value of about $60-80 \mathrm{~h}^{-1} \mathrm{Mpc}$. This shows their complicated structure with subsystems of large curvature. The value of $H_{3}$ is largest in the case of the supercluster SCL9, which is the longest supercluster with the largest number of isolated clumps or cores in it. In the case of other observed superclusters the value of $H_{3}$ decreases when we increase the mass fraction and move into the central parts of the superclusters. This is additional evidence that these superclusters are less clumpy than SCL9 and SCL126. At mass fractions $m_{\mathrm{f}} \approx 0.5$ the value of $\mathrm{H}_{3}$ for these superclusters is less than $20 \mathrm{~h}^{-1} \mathrm{Mpc}$.

Figure 3 (lower panels) shows the shapefinders $H_{1}-H_{3}$ for simulated superclusters. We see that the shapefinder $H_{1}$ (thickness) for the simulated superclusters has values close to those for observed superclusters, but with a much smaller scatter. The widths of the simulated superclusters $\left(\mathrm{H}_{2}\right)$ have values intermediate between those for the observed superclusters SCL9 and SCL126, and for other observed superclusters. Again, the scatter of these values is very small.

The shapefinder $\mathrm{H}_{3}$ (length) shows a rather different picture. The curve for the simulated supercluster M2 is quite similar to that of the observed supercluster SCL9; $\mathrm{H}_{3}$ for the superclusters M3 and M4 are close to those of SCL126. The length of the shortest simulated supercluster M1 is still greater than the length of the shortest observed superclusters, SCL10, SCL88, and the Local Supercluster. The large values of $\mathrm{H}_{3}$ at intermediate mass fractions, $m_{\mathrm{f}} \approx 0.5$ (30 and $90 h^{-1} \mathrm{Mpc}$ ) indicate the presence of substructures in superclusters with high values of the curvature $C$.

In summary, we show how to quantify the thickness, width and length of a supercluster using the shapefinders $H_{1}-H_{3}$ (Table 2 and Fig. 3). Our calculations show that the largest difference between the shapefinders $H_{1}$ to $H_{3}$ for real and simulated superclusters is that in the case of real data, the scatter of the values of these shapefinders is much larger than in the case of model superclusters.

\subsubsection{Shapefinders $K_{1}$ and $K_{2}$}

Next we study the shapefinders $K_{1}$ and $K_{2}$ for the richest superclusters (Fig. 4 and Table 2). $K_{1}$ is defined by the thickness $H_{1}$ and the width $\mathrm{H}_{2}$, this characterizes the planarity of the superclusters; $\mathrm{K}_{2}$ is calculated from the width $\mathrm{H}_{2}$ and the length $\mathrm{H}_{3}$ which characterizes the filamentarity of superclusters.

In the upper panels of Fig. 4 we present the planarity and the filamentarity for observed superclusters. The values of the planarity $K_{1}$ for the full superclusters (the mass fraction $m_{\mathrm{f}}=0$ ) are $0.10-0.15$ for the richest superclusters, SCL126 and SCL9, and about 0.05 for other observed superclusters. As the mass fraction increases and only the higher density parts contribute to superclusters, the values of the planarity $K_{1}$ start to decrease. For the supercluster SCL126 the $K_{1}$ curve has a small minimum at the mass fraction $m_{\mathrm{f}} \approx 0.4$; this is the mass fraction value at which the value of the fourth Minkowski functional $V_{3}$ starts to increase. In all rich superclusters, the value of $K_{1}$ for the core regions becomes negative. This shows that at very high mass fractions, which include only the central regions of superclusters, the isodensity surfaces have complex shapes, different from the heuristic classification based on convex ellipsoids as described above.

The values of the filamentarity for observed superclusters, $K_{2}$, have much larger scatter than the values of the planarity, $K_{1}$. For the richest superclusters, SCL126 and SCL9, $K_{2} \approx 0.2-0.3$, the other observed superclusters have smaller $K_{2}$ (at the mass fraction $m_{\mathrm{f}}=0$ ). The central parts of these superclusters (at high values of mass fractions) are more filamentarity than the full superclusters. The Virgo supercluster V20 has a different shape, for this supercluster the the filamentarity $K 2$ decreases for $m_{\mathrm{f}}<0.2$.

In the case of simulated superclusters, the filamentarities $K_{2}$ have a smaller scatter than those for the observed superclusters. The simulated supercluster M2 has the largest value of $K_{2}$ in our sample, $K_{2} \approx 0.6$, other simulated superclusters have $K_{2} \approx 0.3-0.5$, similar to the observed superclusters SCL126 and SCL9 (for the whole supercluster).

In earlier studies the shapes of superclusters have been characterized using the ratio $K_{1} / K_{2}$ for the full superclusters (Basilakos et al. 2001; Basilakos 2003). We plot this ratio for the whole mass fraction interval in Fig. 4, right panels. This figure shows that the ratios $K_{1} / K_{2}$ for the observed and simulated superclusters are quite similar, having values of about $0.25-0.4$. This shows a high degree of filamentarity in the case of the whole superclusters. The ratios $K_{1} / K_{2}$ slowly decrease, as we increase the mass fraction and move to central regions of superclusters. Exceptions are the observed supercluster SCL10 with the highest values of $K_{1} / K_{2}$ at intermediate mass fractions, and the Virgo supercluster V20 for which this ratio changes strongly.

Figure 4 (right panels) and Table 2 show that the model supercluster M2 has the largest ratio $K_{1} / K_{2}$ among the superclusters under study - this is the most planar supercluster which also has the largest number of clumps in it.

The information about the shapes of superclusters can be best described by their morphological signature, the path in the shapefinder $K_{1}-K_{2}$ plane for varying $m_{\mathrm{f}}$ (Fig. 2, right panels), both for the observed and model superclusters. To show which part of the shape plane corresponds to the whole supercluster, we mark with triangles the values of $K_{1}, K_{2}$ at the mass fraction $m_{\mathrm{f}}=0$ for the superclusters SCL126 and M1. We also mark with circles the values of $K_{1}, K_{2}$ at the mass fraction corresponding to the maximum value of the fourth Minkowski functional $V_{3}$ (Table 2). As explained in Appendix B, we restrict the $m_{\mathrm{f}}$ from below, starting the curves from $m_{\mathrm{f}}=0.01$. This is done to eliminate the influence of the slight non-isotropy of the $B_{3}$ kernel at low densities.

In the shapefinder plane $K_{1}-K_{2}$, the observed superclusters SCL126 and SCL9, and the simulated superclusters have similar trajectories. As we change the mass fraction, the $K_{1}-K_{2}$ shapefinder path moves from low $K_{1}$ and $K_{2}$ values (this corresponds to the whole supercluster and low mass fractions) to the upper left region with higher $K_{2}$ and smaller $K_{1}$ (high mass fractions, the core of the supercluster). At first, as the mass fraction increases, the value of the shapefinder $K_{1}$ (the indicator of planarity) almost does not change, but the value of the shapefinder $K_{2}$ (the indicator of filamentarity) increases, in accordance with Fig. 4. At a certain mass fraction the value of the shapefinder $K_{2}$ reaches its maximum value. As we futher increase the value of the mass fraction (and move to higher densities, into the cores of superclusters), the value of the shapefinder $K_{2}$ changes a little, but the value of the shapefinder $K_{1}$ decreases.

We see that the richest superclusters have a distinct signature in the shapefinder $K_{1}-K_{2}$ plane - a characteristic curve which describes the typical morphology of superclusters. This signature is characterized by a rising path with a small fixed positive $K_{1}$, a plateau at the maximum value of $K_{2}$, and a descending branch at a small fixed negative value of $K_{1}$. In Appendix A we show that this curve is characteristic of multi-branching filaments.

In summary, superclusters are extended 3-dimensional objects composed of multi-branched filaments. Among the 

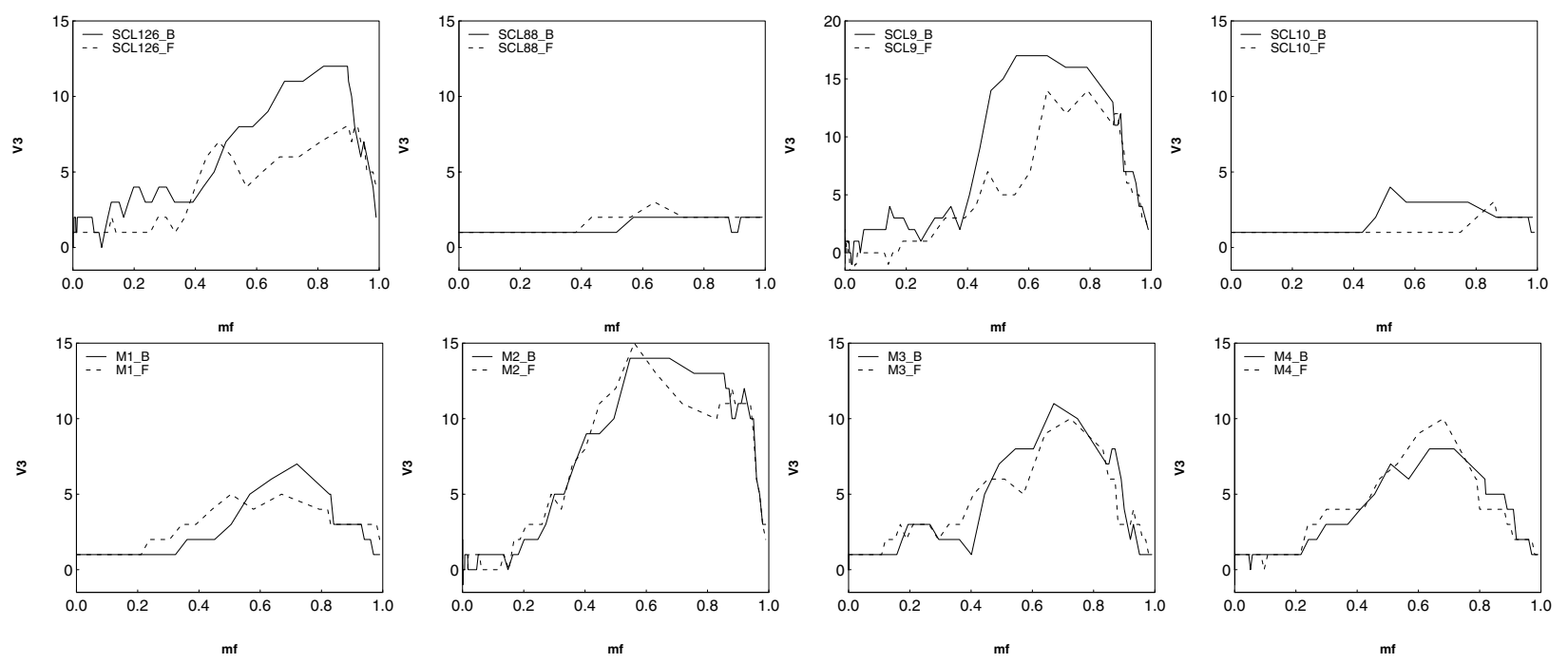

Fig. 5. The fourth Minkowski functional $V_{3}$ for the bright (B, $\left.M \leq-20.0\right)$ and faint (F, $\left.M>-20.0\right)$ galaxies of the observed superclusters $(u p p e r$ panels, from left to right: SCL126, SCL88, SCL9 and SCL10) and for the Millennium simulation (lower panels, from left to right: M1, M2, M3, M4).

observed superclusters, there is a large scatter of the different curves in the $K_{1}-K_{2}$ plane. We find two main types of behavior. In the $K_{1}-K_{2}$ shapefinder plane the morphology of rich superclusters is described by a curve (morphological signature), which is characteristic of multi-branching filaments. In the case of the Virgo supercluster we see how the Minkowski functionals and shapefinders describe a compact supercluster with one central cluster and accompanying filaments, where poorer clusters and groups of galaxies reside. The morphological signature for the Virgo supercluster is characteristic of a spider - a supercluster with one central body, surrounded by filaments (see Appendix A).

\subsection{Distribution of bright and faint galaxies}

As a further application of Minkowski functionals we investigate the distribution of bright and faint galaxies in rich superclusters. We divide galaxies into populations of bright and faint galaxies using a bright/faint galaxy limit $M_{\mathrm{b} j}=-20.0$. The same division was used in Paper III (Einasto et al. 2007c) to study the distribution of bright and faint galaxies in superclusters. Next we calculate the Minkowski functionals separately for these two populations of galaxies, for both real and simulated superclusters.

The fourth Minkowski functional, $V_{3}$, for the bright and faint galaxies in the observed and simulated superclusters is shown in Fig. 5. We see that there are large differences of the fourth Minkowski functional for these populations in observed superclusters. This Minkowski functional characterizes the clumpiness of superclusters. In the supercluster SCL126 a high level of clumpiness is observed over a large range of mass fractions, both for the bright and faint galaxies. For the bright galaxies $V_{3}$ reaches a value of about 10 , while for the faint galaxies the value of $V_{3}$ remains about 5. In the supercluster SCL9 the clumpiness of both the bright and faint galaxies peaks at a relatively high value of the mass fraction, but for the bright galaxies in a broader mass fraction range. In the superclusters SCL88 and SCL10 the clumpiness is very low, again for galaxies of both brightness classes. The fact that the values of $V_{3}$ for the bright galaxies are larger than for the faint galaxies shows that the bright galaxies are located in numerous clumps or cores while the fainter galaxies form a less clumpy population around them. This is a reflection of the well-known luminosity segregation effect. For early determinations of this relation, we refer to Hamilton (1988) and Einasto (1991); the latest study about this segregation is by Park et al. (2007), see also references in Paper RII.

In contrast, the values of $V_{3}$ for the bright and faint galaxies in simulated superclusters differ less than in the case of the observed superclusters. Therefore, the clumpiness of the bright and faint galaxies in model superclusters is rather similar. The reason for this difference between the real and model superclusters is not yet clear; one possible explanation is that the luminositydensity correlation is not well modeled. In Paper I we showed that, on large scales, the luminosity-density relation is built into the Millennium Simulation galaxy sample. However, at small scales there are large differences of clumpiness between these samples; this represents the largest difference between the real and simulated galaxy populations found in this paper.

\section{Discussion}

\subsection{Shapes and sizes of superclusters}

To characterize the shape of an object Sahni et al. (1998), Sheth et al. (2003) and Shandarin et al. (2004) have investigated the morphology of simulated superclusters and voids using the Minkowski functionals and shapefinders. They studied, among others, the largest (percolating) supercluster, and showed that according to shapefinders, this system is filamentary. Sheth et al. (2003) plotted the morphology of the largest (percolating) supercluster in the shapefinder $K_{1}-K_{2}$ plane for a limited interval of threshold densities. In this paper the shapefinder $H_{3}=C / 4 \pi(G+1)(G$ is the genus $)$ is defined differently from their earlier definition that we use $\left(H_{3}=C / 4 \pi\right)$; the first definition gives length per clump). However, if we recalculate our shapefinder in the same way as Sheth et al. (2003), we get rather similar shaped curves as their Fig. 18.

The shapes and sizes of the observed superclusters were studied by Basilakos et al. (2001), Kolokotronis et al. (2002), and by Basilakos (2003), and those of LCDM superclusters by Basilakos et al. (2006) using Minkowski functionals and shapefinders for the density field, which was smoothed with a Gaussian kernel. Kolokotronis et al. (2002) calculated the 
shapefinders $K_{1}, K_{2}$, and their ratio $K_{1} / K_{2}$ for Abell superclusters and showed that about $50 \%$ of all superclusters have the ratio $K_{1} / K_{2}<1$ as is typical for filaments. They also showed that the ratio $K_{1} / K_{2}$ is larger for poor superclusters, which are typically planar structures (pancakes), and smaller for rich superclusters, which are more filamentary. This also agrees with our study which showed that the richest superclusters are multibranching filaments. Similarly, Basilakos (2003) showed that at least $70 \%$ of superclusters from their SDSS supercluster catalog are filamentary (the shapefinder $K_{1} / K_{2}<1$ ). They showed also that in models, filamentary superclusters dominate.

All these studies concern only the "outer" shapes of superclusters and do not treat their substructure. We expand this approach by using the Minkowski functionals and shapefinders to analyze the full density distribution in superclusters, at all density levels.

The Local Supercluster, as the closest and best studied supercluster, serves as a supercluster template for poor superclusters, which are the most numerous superclusters in our catalog. As seen in the morphology figures, its morphology is still different from that of other superclusters in the present selection (see, especially, the $K_{1} / K_{2}$ ratio and its morphological signature in the $K_{1}-K_{2}$ plane).

\subsection{The peculiar supercluster SCL126}

One of the two richest superclusters from the $2 \mathrm{dF}$ survey catalog is the supercluster SCL126 in the Northern sky. In the $K_{1}-K_{2}$ shapefinder plane this supercluster is similar to a multibranching filament. The $V_{3}$ curve for SCL126 has peaks at very high mass fractions $\left(m_{\mathrm{f}}>0.95\right)-$ an indication of a high density compact core. In Einasto et al. (2003c) we showed that the core region of this supercluster contains several Abell clusters, which are also X-ray clusters. This region is about $10 h^{-1} \mathrm{Mpc}$. This supercluster is located almost perpendicularly to the line-of-sight (Jaaniste et al. 1998; Einasto et al. 2003c). In Paper RII we shall show that the fraction of star-forming galaxies, especially in the core region, in this supercluster is lower than in the supercluster SCL9. A possible interpretation of these findings is that this supercluster started to form earlier than the supercluster SCL9.

The Minkowski functionals and shapefinders indicate that this supercluster resembles a rich filament with several branches, and is less clumpy than the other richest superclusters (SCL9, and simulated superclusters). This interpretation agrees with the description of this supercluster as a wall in other papers (the Sloan Great Wall, Hoyle et al. 2002; Vogeley et al. 2004; Gott et al. 2005, 2006; Nichol et al. 2006). This supercluster affects the measurements of the correlation function (Croton et al. 2004), and the genus and Minkowski functionals of the SDSS and $2 \mathrm{dF}$ redshift surveys (Park et al. 2005; Saar et al. 2007). The "meatball" shift in the measurements of the topology in the SDSS data is partly due to this supercluster (Gott et al. 2006). Gott et al. conclude that $N$-body simulations with very large volume and more power at large scales are needed to model such structures more accurately than present simulations. Similar conclusions were reached by Einasto et al. (2006).

\section{Conclusions}

We used a catalog of superclusters of galaxies for the $2 \mathrm{dF}$ Galaxy Redshift Survey and a catalog of model superclusters from the Millennium Simulation to study the morphology and internal structure of the richest superclusters. Our main conclusions are the following.
- We show how to quantify the morphology of superclusters using the Minkowski functionals $V_{0}-V_{3}$ and shapefinders $H_{1}-H_{3}$ and $K_{1}$ and $K_{2}$.

- We find that the fourth Minkowski functional $V_{3}$ describes the clumpiness of superclusters.

- The value of $V_{3}$ indicates that the supercluster SCL126 resembles a multibranching filament, while the supercluster SCL9 can be described as a collection of spiders (a multispider), consisting of a large number of cores connected by relatively thin filaments. Simulated superclusters have the $V_{3}\left(m_{\mathrm{f}}\right)$ curves which differ from those for observed superclusters. The maximum values of the fourth Minkowski functional $V_{3}$ for the richest superclusters from observations and from models (SCL9 and M2) coincide $\left(V_{3}^{\max }=15\right)$, but the shapes of the $V_{3}$ curves for these superclusters are different, indicating that the structure of these superclusters differs.

- The $V_{3}\left(m_{\mathrm{f}}\right)$ curve for SCL126 has peaks at very high mass fractions $\left(m_{\mathrm{f}}>0.95\right)-$ an indication of a high density compact core.

- We show, using empirical geometrical models, that the trajectory traced by the supercluster when we change the mass fraction, forms a curve in the $K_{1}-K_{2}$ plane (the morphological signature), which is characteristic of multibranching filaments. For the Local Supercluster, this curve is characteristic of a system with one central cluster sprouting filaments (the spider type).

- The Minkowski functionals and shapefinders for observed rich superclusters have a much larger scatter than those for simulated superclusters. The selected rich Millennium superclusters show very similar morphological scaling relations $\left(H_{1}\left(m_{\mathrm{f}}\right), H_{2}\left(m_{\mathrm{f}}\right), K_{1}\left(m_{\mathrm{f}}\right), K_{1} / K_{2}\left(m_{\mathrm{f}}\right)\right)$, while these curves vary considerably for the observed superclusters. This shows that the shapes of observed superclusters are more diverse than the shapes of simulated superclusters.

- The values of the fourth Minkowski functional $V_{3}$ show that the clumpiness of real superclusters, for galaxies of different luminosity, has a much larger scatter than the clumpiness of model superclusters.

This is an indication that the fine structure of superclusters (their clumpiness) as expressed by the fourth Minkowski functional $V_{3}$ for bright and faint galaxies in real and simulated superclusters is different, i.e. the model does not yet explain all the features of observed superclusters.

The present analysis supplements previous work, adding more details and using the Minkowski functionals in a novel way. In summary, different methods describe together many aspects of the morphology of superclusters - their sizes, shapes, volumes, compactness and clumpiness, giving an overall picture of their morphology.

The aim of the present paper was to study the morphology of the richest superclusters from the 2dFGRS in detail, and to compare it with that of the richest superclusters from the Millennium Simulation. However, all our superclusters are covered only partly by the $2 \mathrm{dFGRS}$. Therefore, the next step in our analysis is to use the SDSS data to generate supercluster catalogs and to study their morphology. In particular, the region of the supercluster SCL126 is covered by both surveys, therefore giving us a good chance for the comparison of the properties of this supercluster from both data sets. Using a larger sample will also increase the number of superclusters and allow us to obtain statistically significant results. In the present work we studied 
only the richest superclusters, so the results we obtained have only a heuristic nature.

In addition, the volume covered by the Millennium Simulation may be too small to include a large number (and a large morphological variety) of very rich superclusters. Thus we started a study of superclusters from the Hubble Volume simulation, which covers the largest volume at present.

Acknowledgements. We are pleased to thank the 2dFGRS Team for the publicly available data releases. We thank Dr. Tõnu Viik for helpful suggestions and S. Paredes for help with one of the figures. We thank the referee, Dr. M. Vogeley, for constructive comments. The present study was supported by the Estonian Science Foundation grants Nos. 6104 and 7146, and by the Estonian Ministry for Education and Science research project TO 0060058S98. This work has also been supported by the University of Valencia through a visiting professorship for Enn Saar and by the Spanish MEC project AYA2006-14056 (including FEDER). J.E. thanks Astrophysikalisches Institut Potsdam (using DFG-grant 436 EST 17/4/06), and the Aspen Center for Physics for hospitality, where part of this study was performed. PH and PN were supported by Planck science in Metsähovi, Academy of Finland. In this paper we made use of R, a language for data analysis and graphics (Ihaka \& Gentleman 1996).

\section{Appendix A: Morphological templates}

While shapefinders have been used in a number of papers, their meaning needs clarification. In the original paper (Sahni et al. 1998) they were illustrated by calculating them for regular geometric bodies (ellipsoids and tori). The superclusters we find from observations are more complex, frequently branching structures, and kernel estimators give us, as a rule, corrugated density isosurfaces. So, in order to understand better the shapefinders, we calculate them first for a well-known household object, a common kitchen table, and then for several possible supercluster templates.

We build the table by adding layers of different density to a skeleton, consisting of a plate of the size of $40 \times 100$ (grid units), and of four legs (rods) of the length of 60, which join the plate perpendicularly at its corners. We use the parabolic density law:

$\varrho(r)=1-r^{2} / R^{2}, \quad r \leq R ; \varrho(r)=0, \quad$ otherwise,

where $r$ is the nearest distance from a point to the skeleton, and we have taken the limiting distance $R=10$ grid units. This is a rather thick table with thick legs; the reason being follows. First, as we use Crofton formulae to calculate the Minkowski functionals, we have to ensure that our table occupies a generic position in space (the specific weights we use are obtained by assuming statistical isotropy of the isodensity surfaces, see Schmalzing $\&$ Buchert (1997). We do this by aligning the normal to the plate in the direction $\boldsymbol{n}=(1 / \sqrt{3}, 1 / \sqrt{3}, 1 / \sqrt{3})$. As the plate is inclined with the respect to the coordinate grid, the isosurfaces are inevitably slightly jagged, and large $R$ makes them smoother. When following the density isosurfaces inwards, towards larger $m_{\mathrm{f}}$, we find another effect - because of the inclination of the skeleton the high density regions on the grid break up into a large number of isolated clumps around grid points. So we present the shapefinders only for mass fractions $m_{\mathrm{f}} \leq 0.93$ (up to the break-up). The shapefinders are given in Fig. A.1, for the full table, for the plate, for a single leg and for four legs together.

Let us start with the first shapefinder, the thickness $H_{1}$. We know by construction that the true thickness is $2 R=20$ at the outer edges of the table, and reaches 0 inside. The figure shows that $H_{1}$ almost finds the true thickness for the plate, is slightly lower for the $\operatorname{leg}(\mathrm{s})$, starting from 13, and runs in between those two relations for the full table.

The second shapefinder, $\mathrm{H}_{2}$, should give the width of the table. For the plate it should be $40+2 R=60$ at low densities and 40 at high densities. As we see, it runs from about 33 to 22 , closer to the half-width of the plate; this is logical, considering that the shapefinders are normalized requesting that the "width" of a sphere should equal its radius, not diameter. But a similar normalization is used for the thickness above, which, however, finds the full thickness. The width of the $\operatorname{leg}(\mathrm{s})$ is equal to their thickness, $H_{1}=H_{2}-$ the legs are cylinders. And the "width" (half-width, to be more precise) of the table, the combination of the above details, is closer to the widths of the legs; these four appendices dominate the width shapefinder for the full table.

The third shapefinder $\mathrm{H}_{3}$ should give the length of the objects. It starts from about 47 for the plate, and from 28 for a leg. Here, finally, the shapefinder for four legs is four times that for a single leg, as it should be. But these values show that $H_{3}$ really gives the half-length of the object, because of the normalization. And the half-length of the full table is larger than any of its details, but does not equal their sum. Moreover, while the lengths of the details approach those of their skeletons, the length of the full table, which is also reduced to its skeleton for $m_{\mathrm{f}} \rightarrow 1$, stays constant for all values of $m_{\mathrm{f}}$, from the "fat" table to the skeleton.

The "second-order" shapefinder, the planarity $K_{1}$, is small (about 0.06 ) for the $\operatorname{leg}(\mathrm{s})$, runs from 0.27 (thick plate) to 0.8 (fat skeleton) for the plate, and from 0.18 to 0.6 for the full table. These numbers are as expected, only the maximum planarity for the plate seems to be too small; but, as explained above, this might be simply a non-aligned grid effect.

The filamentarity $K_{2}$ is smallest for the plate, ranging from 0.17 to 0.27 . It is not too large for a single leg (from 0.3 to 0.7 , the legs are thick), but ranges from 0.76 to 0.92 for the collection of four legs. The filamentarity of the full table is dominated by that of the legs; it ranges from 0.64 to 0.85 ; a kitchen table is basically a filamentary object.

The planarity/filamentarity ratio $K_{1} / K_{2}$ that is frequently used, runs from 0.2 to about 0.1 for a single leg, and is lower than 0.07 for the collection of four legs. It is high (larger than 1.5 ) for the plate, but pretty low (between 0.5 and 0.7 ) for the full table; another indication that legs define the morphology of a table. This curve is quite jagged, and the reason for that is clear - the "first order" shapefinders $H_{i}$ are ratios of Minkowski functionals, the "second order" shapefinders $K_{i}$ are ratios of these ratios, and the ratio $K_{1} / K_{2}$ becomes noisy. This is also seen in the case of our observed superclusters (see Fig. 4), especially for small values of $K_{2}$, and may partly explain the large scatter of the ratio $K_{1} / K_{2}$. Because of this, we advocate the use of the morphological signature (see below).

We can also look at the morphological signatures (the trajectories in the $K_{1}-K_{2}$ plane, parameterized by $m_{\mathrm{f}}$ ) of the table and its details (Fig. A.1, lower right panel). We limit the mass fraction range for this figure also from below, using only $m_{\mathrm{f}} \in$ $[0.01,0.94]$, as done in the main paper (see also Appendix B). This choice eliminates grid effects at low and high densities, where our $B_{3}$ kernel is not isotropic. The Crofton formulae we use assume isotropy, so it is safer to avoid these densities.

Ignoring the jagged nature of the curves (these can be eliminated, in principle, by constructing direct estimates for the shapefinders $K_{1}$ and $K_{2}$ ) we see that the details and the table have clear and distinct signatures. The signature for the plate lies in the planar region, that for the leg(s) in the region describing filaments (and four legs together are clearly more filamentary than a single leg). The full table is both planar $K_{1} \in[0.2,0.7]$ and filamentary $K_{2} \in[0.65,0.9]$, but clearly rather filamentary than planar $\left(K_{2}>K_{1}\right)$.

The kitchen table was an example of a smooth density distribution. The density of our superclusters is obtained by summing 

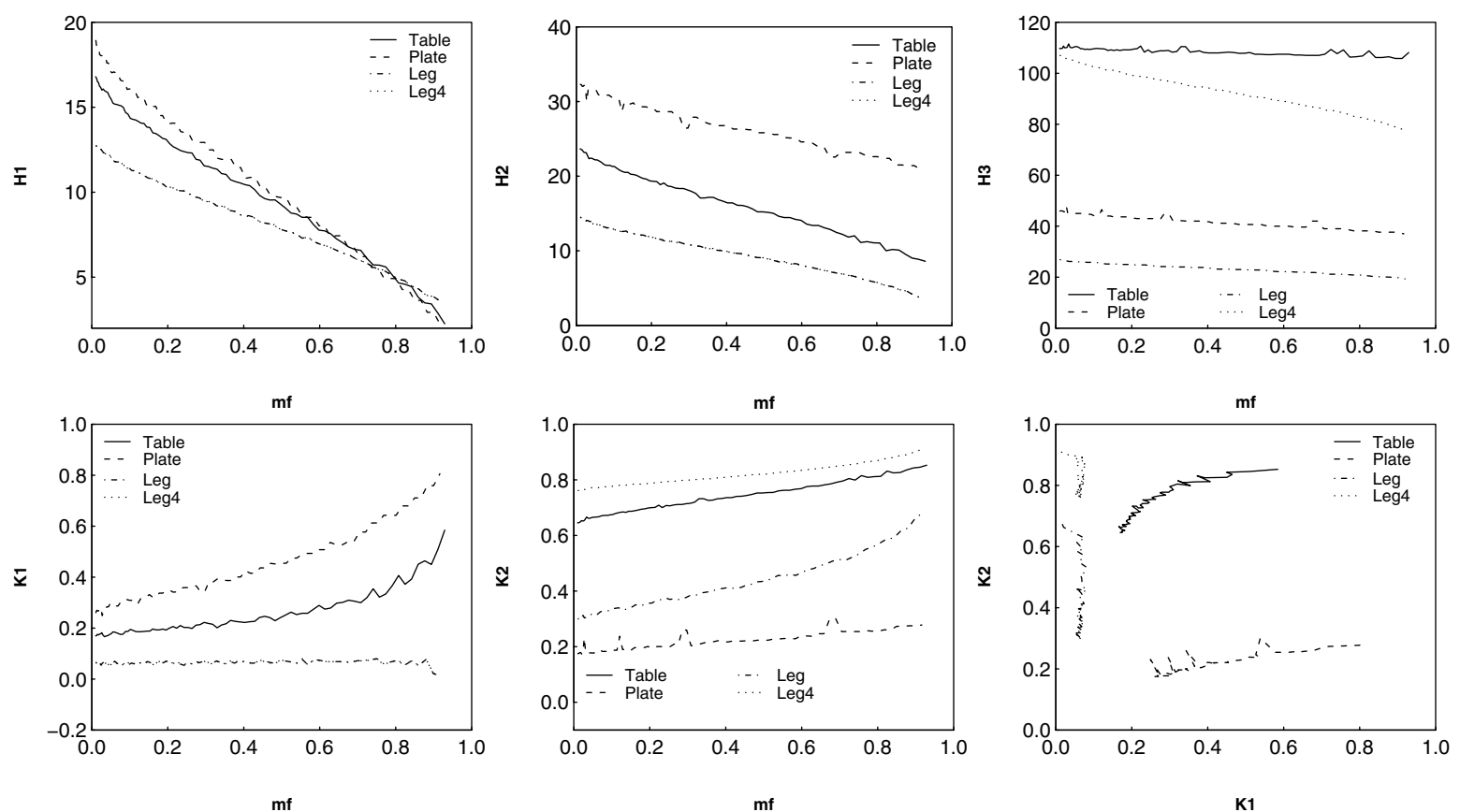

Fig. A.1. The shapefinders $H_{1}$ (thickness), $H_{2}$ (width), $H_{3}$ (length), upper row, and $K_{1}$ (planarity), $K_{2}$ (filamentarity), and the morphological signature for the table and its details.
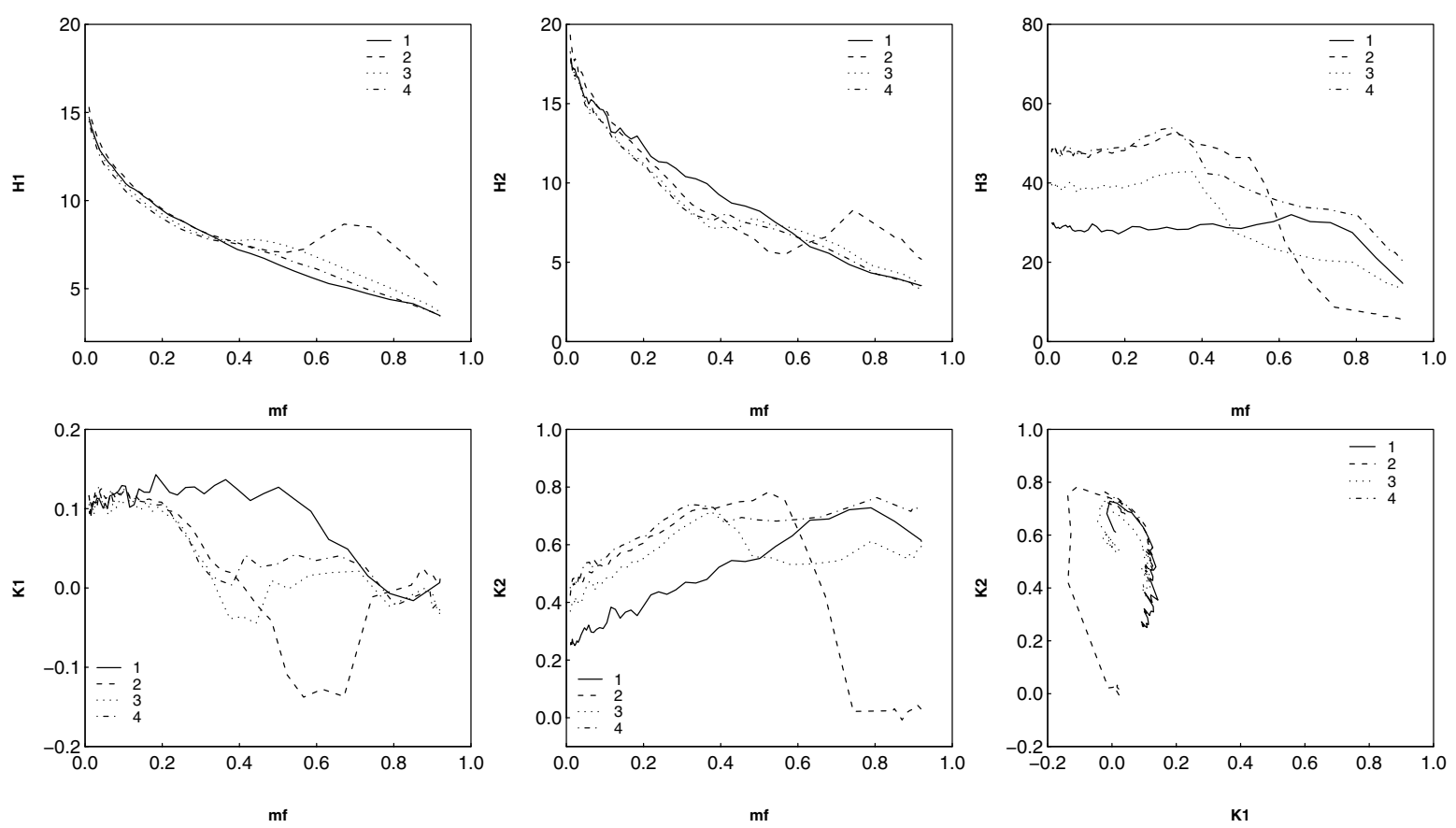

Fig. A.2. The shapefinders $H_{1}$ (thickness), $H_{2}$ (width), $H_{3}$ (length), upper row, and $K_{1}$ (planarity), $K_{2}$ (filamentarity), and the morphological signature (lower row) for different templates. The labels are: 1 - filament, 2 - spider, 3 - Jacob's staff, and 4 - Cross of Lorraine (Karelian reindeer).

together almost isotropic kernels around galaxy locations. This results in a much more patchy density distribution; on the other hand, it automatically guarantees statistical isotropy of the density isolevels. In order to better understand the shapefinders of the observed superclusters, we calculate them for a number of simple templates which resemble the superclusters. These are: 1) a single long filament, 2) a spider (two long filaments, crossing, with a small cube at the center), 3) Jacob's staff or ballestina (an astronomical instrument from middle ages for measuring angular distances; a long staff with a perpendicular smaller staff), and 4) the Cross of Lorraine (similar to the Jacob's staff, but with two perpendicular staves, with a spherical blob at the center). The last configuration resembles also ancient petroglyphs from Karelia, depicting thunder and reindeer. The dimensions are: 100 (grid units) for the filament (and staff) length, 40 for the small staff length, 10 for the radius of the blob, and the effective width of the density kernel is 8 (total width 16). The corresponding shapefinders are shown in Fig. A.2.

As we see, the shapefinders are smoother, and clearly distinct for different morphologies. The complex (corrugated) nature of 
the isodensity surfaces causes local minima for the lengths $H_{i}$ at certain mass fractions (in the thickness and in the width of the spider). Also, the length shapefinder $\mathrm{H}_{3}$ almost does not change for a wide initial interval of mass fractions, while the isodensity surfaces contract towards the skeletons of our templates. As this length is defined both by the isodensity surface area and its curvature, a simple behavior cannot always be expected.

A new feature here is that the "second order" shapefinder $K_{1}$ acquires negative values for specific density isolevels. This is possible, as the inequalities $H_{1} \leq H_{2} \leq H_{3}$ which are usually assumed, are valid only for convex bodies. The isolevels for kernel-estimated densities are usually corrugated, and the real limits for $K_{i}$ are: $K_{i} \in[-1,1]$. This may prevent the use of the usual morphological index $K_{1} / K_{2}$; it is better to use the "morphological signature" $K_{2}$ versus $K_{1}$ instead.

Looking at Fig. A. 2 we see that there are two morphologies which resemble those of the observed superclusters. The "spider" imitates poor Virgo-type superclusters, although our model spider has a large value $K_{2}$, rich superclusters (as SCL126, see Fig. 2) are best represented by the Cross of Lorraine, a multibranching filament. Adding a central body to that system improves the similarity even more.

We do not attempt to fit supercluster models to observations in this paper; this will need a well-parameterized basic model. However, we see that a good starting point for such a project would be the two models listed above.

\section{Appendix B: Kernel densities}

When studying the morphology of superclusters of galaxies, a necessary step is to convert the spatial positions of galaxies into spatial densities. The standard approach is to use kernel densities (see, e.g., Silverman 1986):

$\varrho(x)=\sum_{i} K\left(\boldsymbol{x}-\boldsymbol{x}_{i} ; h\right) m_{i}$,

where the sum is over all galaxies, $\boldsymbol{x}_{i}$ are the coordinates of the $i$ th galaxy, and $m_{i}$ is its mass (or luminosity, if we are estimating luminosity densities; for number densities we set $m_{i} \equiv 1$ ). The function $K(\boldsymbol{x} ; h)$ is the kernel of the width $h$, and a suitable choice of the kernel determines the quality of the density estimate. If the kernels widths depend either on the coordinate $\boldsymbol{x}$ or on the position of the $i$ th galaxy, the densities are adaptive. We are using constant width kernels in this paper, defining superclusters as density enhancements of a common scale (the typical density scale of about $8 h \mathrm{Mpc}^{-1}$ ). Kernels have to be normalized and symmetrical:

$\int K(\boldsymbol{x} ; h) \mathrm{d} V=1, \quad \int \boldsymbol{x} K(\boldsymbol{x} ; h) \mathrm{d} V=0$.

Statisticians classify kernels by their MSE (minimal standard error); the best kernel is the Epanechnikov kernel $K_{\mathrm{E}}$ (see Silverman 1986):

$K_{\mathrm{E}}(\boldsymbol{x} ; h)=\frac{15}{8 \pi h^{3}}\left(1-\boldsymbol{x}^{2} / h^{2}\right), \quad \boldsymbol{x}^{2} \leq h^{2}, \quad 0 \quad$ otherwise

(all the kernel expressions in this appendix are for 3D kernels).

In cosmology, the Gaussian kernel $K_{\mathrm{G}}$ has been the most popular:

$K_{\mathrm{G}}(\boldsymbol{x} ; h)=\frac{1}{(2 \pi)^{3 / 2} h^{3}} \exp \left(-\boldsymbol{x}^{2} / 2 h^{2}\right)$.

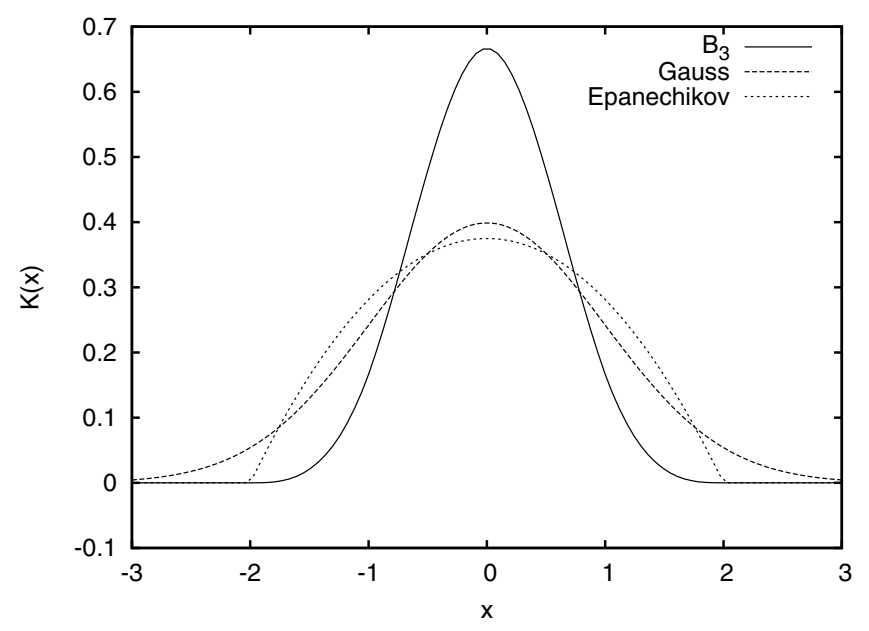

Fig. B.1. Three popular kernels for density estimation - Epanechnikov, Gaussian, and the $B_{3}$ box spline. All the profiles have approximately the same extent (the Gaussian profile width $h=1$, the $B_{3}$ profile is given for $N=1$, and for the Epanechnikov profile $h=2$. All profiles are normalized for the $1 \mathrm{D}$ case.

For the usual case, when densities are calculated for a spatial grid, good kernels are generated by box splines $B_{J}$ (usually used in $N$-body mass assignment). Box splines have compact support (they are local), and they are interpolating on a grid:

$\sum_{i} B_{J}(x-i)=1$

for any $x$, and a small number of indices that give non-zero values for $B_{J}(x)$. In this paper we restrict us to the popular $B_{3}$ splines:

$B_{3}(x)=\frac{1}{12}\left[|x-2|^{3}-4|x-1|^{3}+6|x|^{3}-4|x+1|^{3}+|x+2|^{3}\right]$

(this function is different from zero only in the interval $x \in$ $[-2,2]$, see Fig B.1). We define the (one-dimensional) $B_{3}$ box spline kernel of width $h=N$ as

$K_{B}^{(1)}(x ; N)=B_{3}(x / N) / N$.

This kernel preserves the interpolation property (mass conservation) for all kernel widths that are integer multiples of the grid step, $h=N$. The 3D $K_{B}^{(3)}$ box spline kernel we use is given by the direct product of three one-dimensional kernels:

$\left.K_{B}(\boldsymbol{x} ; N) \equiv K_{B}^{(3)}(\boldsymbol{x} ; N)=K_{B}^{(1)}\right)(x ; N) K_{B}^{(1)}(y ; N) K_{B}^{(1)}(z ; N)$,

where $\boldsymbol{x} \equiv\{x, y, z\}$.

These kernels encompass all the "good" and "bad" kernel properties (good and bad for our application).

- First, the Epanechnikov and the $B_{3}$ kernels are both compact, while the Gaussian kernel is infinite and has to be cut off at a fixed radius. This introduces an extra parameter and, what is more important, generates small-amplitude density jumps which may distort the Minkowski functionals (these are extremely sensitive to small-amplitude density details).

- Second, the $B_{3}$ kernel conserves density (it is exactly normalized on a grid). For continuous kernels normalization on a grid is equivalent to simple numerical integration of the profile; the (truncated) Gaussian kernel demands fever grid points for this than the Epanechnikov kernel. Many grid points inside the kernel profile means that kernel widths must 


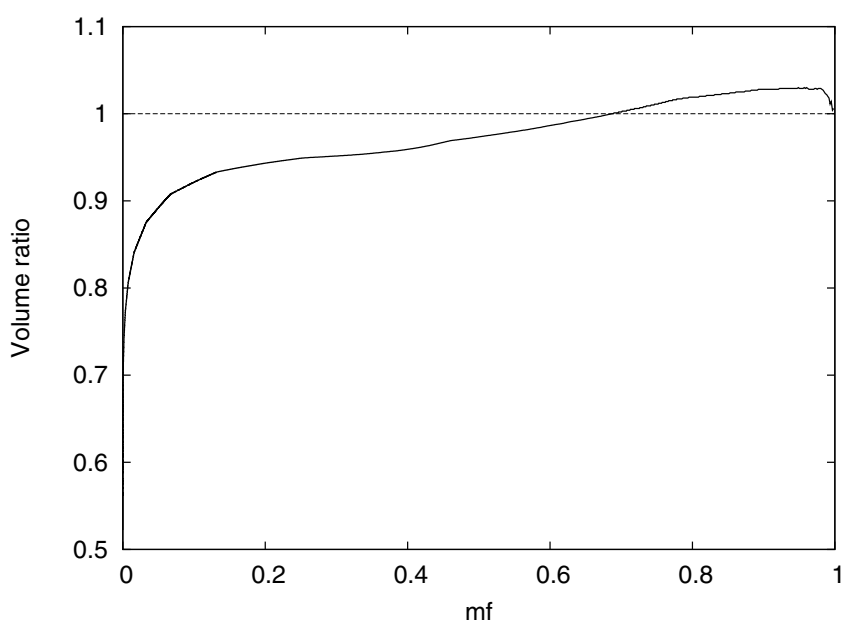

Fig. B.2. Deviation from isotropy for the 3D $B_{3}$ kernel: the ratio of the volume inside an isolevel to the volume of the sphere which cuts coordinate axes at the same point as the isolevel (an inscribed sphere for larger radii, an encompassing sphere for smaller radii). The isolevels are labeled by the mass fraction, the kernel mass outside the isolevel. For an isotropic kernel this ratio should be 1 .

be much larger than the grid step. Bad normalization leads to distortion of the final densities; how well the contribution of a galaxy to the final density is accounted for depends on the location of the galaxy with respect to the grid.

- Third, an important kernel property is its isotropy. The formulae we use to estimate the Minkowski functionals assume average isotropy of the density isosurfaces (their isotropic orientation). Both the Epanechnikov and Gaussian kernels are exactly isotropic, the $3 \mathrm{D} B_{3}$ kernel is not. But this kernel is approximately isotropic to a high degree.

A simple characteristic of isotropy of the kernel isolevels would be the ratio of its surface to the surface of the sphere that cuts coordinate axes at the same point as the isolevel. Alas, surfaces are not easy to calculate, and we replace this ratio by the ratio of the volumes encompassed by these two surfaces; volumes are easy to estimate by Monte-Carlo integration. These ratios are close (e.g., for a cube and the inscribed sphere the volume ratios coincide with the surface ratios). For the $3 \mathrm{D} B_{3}$ kernel, the dependence of this ratio (the sphere volume to the excursion set volume) as a function of the mass fraction (integrated kernel mass outside the isolevel) is shown in Fig. B.2.

As we see, this ratio is close to unity for almost all mass fractions for the $B_{3} \mathrm{kernel}$; considerable deviations can be seen only for small mass fractions. The deviation from isotropy is less than $10 \%$ for $m_{\mathrm{f}} \geq 0.058$. When displaying our results on the morphological descriptors, we used a mass fraction limit for the full supercluster; this is smaller than the value cited above by the ratio of the number of galaxies defining the supercluster limits to the total number of galaxies in the supercluster. To be on the safe side, we deliberately underestimated this ratio and we chose the limit $m_{\mathrm{f}} \geq 0.01$ for the full supercluster.

\section{References}

Abell, G. 1958, ApJS, 3, 211

Abell, G., Corwin, H., \& Olowin, R. 1989, ApJS, 70, 1
Bardelli, S., Zucca, E., Zamorani, G., Moscardini, L., \& Scaramella, R. 2000, MNRAS, 312, 540

Basilakos, S. 2003, MNRAS, 344, 602

Basilakos, S., Plionis, M., \& Rowan-Robinson, M. 2001, MNRAS, 323, 47

Basilakos, S., Plionis, M., Yepes, G., Gottlöber, S., \& Turchaninov, V. 2006, MNRAS, 365, 539

Colless, M. M., Peterson, B. A., Jackson, C. A., et al. 2003 [arXiv: astro-ph/0306581]

Colless, M. M., Dalton, G. B., Maddox, S. J., et al. 2001, MNRAS, 328, 1039

Croton, D. J., Gaztañaga, E., Baugh, C. M., et al. 2004, MNRAS, 352, 1232

Croton, D. J., Farrar, G. R., Norberg, P., et al. 2005, MNRAS, 356, 1155

Croton, D. J., Springel, V., White, S. D. M., et al. 2006, MNRAS, 365, 11

De Propris, R., et al. (2dF GRS Team) 2003, MNRAS, 342, 725

Einasto, M. 1991, MNRAS, 252, 261

Einasto, J., Klypin, A. A., Saar, E., \& Shandarin, S. F. 1984, MNRAS 206, 529

Einasto, M., Einasto, J., Tago, E., Dalton, G., \& Andernach, H. 1994, MNRAS, 269,301

Einasto, M., Tago, E., Jaaniste, J., Einasto, J., \& Andernach, H. 1997, A\&A Suppl., 123, 119

Einasto, M., Einasto, J., Tago, E., Müller, V., \& Andernach, H. 2001, AJ, 122, 2222 (E01)

Einasto, J., Einasto, M., Hütsi, G., et al. 2003a, A\&A, 410, 425 (E03a)

Einasto, J., Hütsi, G., Einasto, M., et al. 2003b, A\&A, 405, 425 (E03b)

Einasto, M., Jaaniste, J., Einasto, J., et al. . 2003c, A\&A, 405, 821

Einasto, J., Einasto, M., Saar, E., et al. 2006 A\&A, 459, L1

Einasto, J., Einasto, M., Tago, E., et al. 2007a, A\&A, 462, 811 (Paper I)

Einasto, J., Einasto, M., Saar, E., et al. 2007b, A\&A, 462, 397 (Paper II)

Einasto, M., Einasto, J., Tago, E., et al. 2007c, A\&A, 464, 815 (Paper III)

Einasto, M., Saar, E., Einasto, J., et al. 2007d, A\&A, submitted, (RII)

Erdogdu, P., Lahav, O., Zaroubi, S., et al. 2004, MNRAS, 352, 939

Fleenor, M. C., Rose, J. A., Christiansen, W. A., et al. 2005, AJ, 130, 957

Gott, J. R., Juric, M., Schleger, D., et al. 2005, ApJ, 624, 463

Gott, J. R., Hambrick, D. C., Vogeley, M. S., et al. 2006 [arXiv: astro-ph/0610762]

Hamilton, A. J. S. 1988, ApJ, 331, L59

Hoyle, F., et al. 2002, ApJ, 580, 663

Ihaka, R., \& Gentleman, R. 1996, J. Computational Graphical Stat., 5, 299

Jaaniste, J., Tago, E., Einasto, M., et al. 1998, A\&A, 336, 35

Kofman, L. A., Einasto, J., \& Linde, A. D. 1987, Nature, 326, 48

Kolokotronis, V., Basilakos, S., \& Plionis, M. 2002, MNRAS, 331, 1020

Lahav, O. 2004, Pub. Astr. Soc. Australia, 21, 404

Lahav, O. 2005, in Nearby Large-Scale Structures and the Zone of Avoidance, ed. A. P. Fairall, \& P. Woudt, ASP Conf. Ser., 329, 3

Madgwick, D. S., Somerville, R., Lahav, O., \& Ellis, R. 2003a, MNRAS, 343, 871

Madgwick, D. S., Hawkins, E., Lahav, O., et al. 2003b, MNRAS, 344, 847

Martínez, V. J., Starck, J.-L., Saar, E., et al. 2005, ApJ, 634, 744

Mobasher, B., Dickinson, M., Ferguson, H. C., et al. 2005, ApJ, 635, 832

Nichol, R. C., Sheth, R. K., Suto, Y., et al. 2006, MNRAS, 368, 150

Ouchi, M., Shimasaku, K., Akiyama, M., et al. 2004, ApJ, 620, L1

Park, C., Choi, Y.-Y., Vogeley, M. S., et al. 2005, ApJ, 633, 11

Park, C., Choi, Y.-Y., Vogeley, M. S., et al. 2007, ApJ, 658, 898

Plionis, M. 2004, in Outskirts of galaxy clusters: intense life in the suburbs, Proc. IAU Colloq., 195, ed. A. Diaferio

Porter, S. C., \& Raychaudhury, S. 2005, MNRAS, 364, 1387

Proust, D., Quintana, H., Carrasco, E. R., et al. 2006, A\&A, 447, 133

Rose, J. A., Gaba, A. E., Christiansen, W. A., et al. 2002, AJ, 123, 1216

Saar, E., Martínez, V. J., Starck, J.-L., \& Donoho, D. L. 2007, MN, 374, 1030

Sahni, V., Sathyaprakash, B. S., \& Shandarin, S. 1998, ApJ, 495, L5

Schechter, P. 1976, ApJ, 203, 297

Schmalzing, J., \& Buchert, T. 1997, ApJ, 482, L1

Sheth, J. V., Sahni, V., Shandarin, S. F., \& Sathyaprakash, B. 2003, MN, 343, 22

Shandarin, S. F., Sheth, J. V., \& Sahni, V. 2004, MN, 353, 162

Silverman, B. W. 1986, Density estimation for statistics and data analysis (Boca Raton: Chapman \& Hall/CRX Press)

Springel, V., White, S. D. M., Jenkins, A., et al. 2005, Nature, 435, 629

Tago, E., Einasto, J., Saar, E., et al. 2006, AN, 327, 365 (T06)

Venemans, B. P., Röttgering, H. J., Overzier, R. A., et al. 2004, A\&A, 424, L17

Vogeley, M. S., Hoyle, F., Rojas, R. R., et al. 2004, in Outskirts of galaxy clusters: intense life in the suburbs, Proc. IAU Colloq., 195, ed. A. Diaferio

Zucca, E., Zamorani, G., Scaramella, R., \& Vettolani, G. 1993, ApJ, 407, 470 